Bull. Nov. Comp. Center, Comp. Science, 38 (2015), 121-150

(C) 2015 NCC Publisher

\title{
Bisimulation for fluid stochastic Petri nets*
}

\author{
I. V. Tarasyuk, P. Buchholz
}

\begin{abstract}
We propose a novel notion of fluid bisimulation equivalence that allows one to compare and reduce the behavior of labeled fluid stochastic Petri nets (LFSPNs) while preserving their discrete and continuous properties. The underlying stochastic model for the discrete part of the LFSPNs is a continuous time Markov chain (CTMC). The performance analysis of the continuous part of the LFSPNs is accomplished via the associated stochastic fluid models. For the fluid bisimulation on the discrete markings of two LFSPNs, we require it to be a (strong) Markovian bisimulation. On the continuous markings, for every pair of Markovian bisimilar discrete markings, the fluid flow rates of the continuous places in the first LFSPN should coincide with those of the corresponding continuous places in the second LFSPN. We prove that the resulting fluid bisimulation equivalence preserves fluid density and distribution, as well as discrete and continuous performance measures.
\end{abstract}

Keywords: labeled fluid stochastic Petri net, continuous time stochastic Petri net, continuous time Markov chain, stochastic fluid model, fluid density and distribution, performance analysis, Markovian bisimulation, fluid bisimulation.

\section{Introduction}

An important scientific problem that has been often addressed in the last decades is the design and analysis of parallel systems, which takes into account both qualitative (functional) and quantitative (timed, probabilistic, stochastic) features of their behavior. The main goal of the research on this topic is the development of models and methods respecting performance requirements to concurrent and distributed systems with time constraints (such as deterministic, nondeterministic and stochastic time delays) to construct, validate and optimize the performability of realistic large-scale applications. A fruitful approach to achieving progress in this direction appeared to be a combined application of the theories of Petri nets, stochastic processes and fluid flow systems to the specification and analysis of such time-dependent systems with inherent behavioral randomicity [17].

In the past, many extensions of stochastic Petri nets (SPNs) [22, 23, 2] have been developed to specify, model, simulate and analyze some particular classes of systems, such as computer systems, communication networks or manufacturing plants. One of the extensions are fluid stochastic Petri nets (FSPNs), capable of modeling hybrid systems that combine continuous state

${ }^{*}$ Partially supported by Deutsche Forschungsgemeinschaft (DFG) under Grant BE 1267/14-1 and Russian Foundation for Basic Research (RFBR) under Grant 14-01-91334. 
variables, corresponding to the fluid levels, with discrete state variables, specifying the token numbers. The continuous part of the FSPNs allows one to represent the fluid level in continuous places and fluid flow along continuous arcs. This part can naturally describe continuous variables in physical systems whose behavior is commonly represented by differential equations. Continuous variables may also be used to describe a macroscopic view of discrete items that appear in large populations, e.g., packets in a computer network, molecules in a chemical reaction or people in a crowd. The discrete part of an FSPN is essentially its underlying SPN, obtained from the FSPN by removing all the fluid-related continuous elements. This part usually models the discrete control of the continuous process. The control may demonstrate some stochastic behavior that captures uncertainty about the detailed system behavior.

FSPNs have been proposed in [26, 9, 29] to model stochastic fluid flow systems $[16,12]$. To analyze FSPNs, simulation, numerical and matrixgeometric methods are widely used $[20,10,13,14,11,21,15]$. The major problem of FSPNs is the high complexity of computing their solution, resulting in huge memory and time requirements while analyzing realistic models. A positive feature of the FSPN formalism is that it hides from a modeler the technical difficulties with solving differential equations for the underlying stochastic processes and that it unifies in one framework the evolution equations for the discrete and continuous parts of systems.

However, as far as we know, neither transition labeling nor behavioral equivalences have been proposed so far for FSPNs. In [27, 28], label equivalence and projected label equivalence have been introduced for Fluid Process Algebra (FPA). FPA is a simple sub-algebra of Grouped PEPA (GPEPA) [18], which is itself a conservative extension of Performance Evaluation Process Algebra (PEPA) [19], obtained by adding fluid semantics with an objective to simplify solving the systems of replicated ordinary differential equations. In [27, 28], it has been proved that projected label equivalence induces a fluid lumpable partition and that both label equivalence and projected label equivalence imply semi-isomorphism (stochastic isomorphism) for a subclass of well-posed models. Nevertheless, the mentioned label equivalences do not respect the action types; hence, they are not behavioral relations.

In this paper, we propose a behavioral relation of fluid bisimulation equivalence that is useful for the comparison and reduction of the behavior of labeled FSPNs (LFSPNs), since it preserves the functionality and performability of their discrete and continuous parts.

For every FSPN, the discrete part of its marking is determined by the natural number of tokens contained in the discrete places. The continuous places of an FSPN are associated with the non-negative real-valued fluid levels that determine the continuous part of the FSPN marking. Thus, FSPNs have a hybrid (discrete-continuous) state space. In each hybrid marking, 
its discrete part has an influence on the continuous part. For more general FSPNs, the reverse dependence is possible as well. As a basic model for constructing LFSPNs, we consider only those FSPNs in which the continuous parts of markings have no influence on the discrete ones, i.e. such that every discrete part determines completely both the set of enabled transitions and the rates of incoming and outgoing arcs for each continuous place $[11,15]$. We also require that the discrete part of LFSPNs should be labeled continuous time stochastic Petri nets (CTSPNs) [22, 23, 2].

We require the fluid bisimulation on the discrete parts of the hybrid markings (called discrete markings) of two LFSPNs to be a standard (strong) Markovian bisimulation. Hence, for each transition firing in an LFSPN, we require a simulation of the firing in the equivalent LFSPN, such that the action labels of the both fired transitions and their overall rates coincide. Thus, our definition of the bisimulation equivalence on the discrete markings of LFSPNs is similar to that of the performance bisimulation equivalences $[6,7]$ on labeled CTSPNs and labeled generalized SPNs (GSPNs) $[22,8,23$, $5,2]$, as well as the strong equivalence from [19] on stochastic process algebra PEPA. All these relations belong to the family of Markovian bisimulation equivalences [3]. As for the continuous parts of the hybrid markings (called continuous markings), we should fix a bijective correspondence between the sets of continuous places of the two LFSPNs, hence, the number of their continuous places should coincide and each continuous place in one LFSPN should have exactly one corresponding continuous place in the other LFSPN. Then we should require that, for every pair of Markovian bisimilar discrete markings, the fluid flow rates of the continuous places in the first LFSPN should coincide with those of the corresponding continuous places in the second LFSPN. Note that in our formal definition of fluid bisimulation, we consider only LFSPNs having a single continuous place, since the definition can be easily extended to the case of several continuous places. We prove that the resulting fluid bisimulation equivalence of LFSPNs preserves the stationary probability distribution of the underlying continuous time Markov chain (CTMC), as well as the stationary fluid buffer empty probability, probability distribution and density for the associated stochastic fluid model (SFM). As a consequence, the equivalence guarantees identity of a number of discrete and continuous performance measures, calculated for the stationary quantitative behavior of the LFSPNs.

The paper is organized as follows. In Section 2, we present the definition and behavior of LFSPNs. Section 3 explores the discrete part of LFSPNs, i.e. the derived labeled CTSPNs and their underlying CTMCs. Section 4 investigates the continuous part of LFSPNs, which is their underlying SFMs. In Section 5, we propose fluid bisimulation equivalence for LFSPNs. Section 6 contains the preservation results for the quantitative behavior of LFSPNs modulo the introduced equivalence. In Section 7 , we give an example of the 
Table 1. Abbreviations used in the paper

\begin{tabular}{|ll|l|}
\hline \multicolumn{2}{|l|}{ Petri nets } & Markov chains \\
SPN & STMC continuous time \\
CTSPN & continuous time stochastic Petri net & Markov chain \\
GSPN & generalized stochastic Petri net & SMC semi-Markov chain \\
FSPN fluid stochastic Petri net & Fluid models \\
LFSPN labeled fluid stochastic Petri net & SFM stochastic fluid model \\
Probability functions & Rate matrices \\
PMF probability mass function & TRM transition rate matrix \\
PDF $\quad$ probability distribution function & FRM fluid rate matrix \\
\hline
\end{tabular}

equivalent LFSPNs that demonstrates how to analyze and compare their stationary behavior and performance measures. Section 8 summarizes the results obtained and outlines research perspectives in this area. To help the reader, we present some important abbreviations from the paper in Table 1.

\section{Basic concepts of LFSPNs}

Let us introduce a class of labeled fluid stochastic Petri nets (LFSPNs), whose transitions are labeled with action names used to specify different system activities. Without labels, LFSPNs are essentially a subclass of FSPNs $[20,11,15]$, so that their discrete part describes a CTSPN $[22,23,2]$. This means that LFSPNs have no inhibitor arcs, priorities and immediate transitions, used in the standard FSPNs, which are the continuous extension of GSPNs. However, in many practical applications, the performance analysis of GSPNs is simplified by transforming them into CTSPNs or reducing their underlying semi-Markov chains into CTMCs (the underlying stochastic processes of CTSPNs) by eliminating vanishing states $[8,23,2]$. Transition labeling in LFSPNs is similar to that in the CTSPNs from [6]. We also suppose that the firing rates of transitions and flow rates of the continuous arcs do not depend on the continuous markings (fluid levels).

Let $\mathbb{N}=\{0,1,2, \ldots\}$ be the set of all natural numbers and $\mathbb{N}_{\geq 1}=$ $\{1,2, \ldots\}$ be the set of all positive natural numbers. Let $\mathbb{R}=(-\infty ; \infty)$ be the set of all real numbers, $\mathbb{R}_{\geq 0}=[0 ; \infty)$ be the set of all non-negative real numbers and $\mathbb{R}_{>0}=(0 ; \infty)$ be the set of all positive real numbers. The set of all row vectors of $n \in \mathbb{N}_{\geq 1}$ elements from a set $X$ is $X^{n}=\left\{\left(x_{1}, \ldots, x_{n}\right) \mid\right.$ $\left.x_{i} \in X(1 \leq i \leq n)\right\}$. The set of all mappings from a set $X$ to a set $Y$ is $Y^{X}=\{f \mid f: X \rightarrow Y\}$. Let Act $=\{a, b, \ldots\}$ be the set of actions.

First, we present a formal definition of LFSPNs.

Definition 1. A labeled fluid stochastic Petri net (LFSPN) is a tuple $N=\left(P_{N}, T_{N}, W_{N}, R_{N}, \Omega_{N}, L_{N}, \mathcal{M}_{N}\right)$, where

- $P_{N}=P d_{N} \uplus P c_{N}$ is a finite set of discrete and continuous places;

- $T_{N}$ is a finite set of transitions, such that $P_{N} \cup T_{N} \neq \emptyset$ and $P_{N} \cap T_{N}=\emptyset$; 
- $W_{N}:\left(P d_{N} \times T_{N}\right) \cup\left(T_{N} \times P d_{N}\right) \rightarrow \mathbb{N}$ is a function providing the weights of discrete arcs between discrete places and transitions;

- $R_{N}:\left(\left(P c_{N} \times T_{N}\right) \cup\left(T_{N} \times P c_{N}\right)\right) \times \mathbb{N}^{\left|P d_{N}\right|} \rightarrow \mathbb{R}_{\geq 0}$ is a function of the flow rates of continuous arcs between continuous places and transitions in a given discrete marking (the markings will be defined later);

- $\Omega_{N}: T_{N} \times \mathbb{N}^{\left|P d_{N}\right|} \rightarrow \mathbb{R}_{>0}$ is the transition rate function associating transitions with rates in a given discrete marking;

- $L_{N}: T_{N} \rightarrow$ Act is the transition labeling function assigning actions to transitions;

- $\mathcal{M}_{N}=\left(M_{N}, \mathbf{0}\right)$, where $M_{N} \in \mathbb{N}^{\left|P d_{N}\right|}$ and $\mathbf{0}$ is a row vector of $\left|P c_{N}\right|$ values 0 , is the initial (discrete-continuous) marking.

Consider in more detail the items from the definition of an LFSPN $N$ above.

Every discrete place $p_{i} \in P d_{N}$ may contain discrete tokens, whose amount is represented by a natural number $M_{i} \in \mathbb{N}\left(1 \leq i \leq\left|P d_{N}\right|\right)$. Each continuous place $q_{j} \in P c_{N}$ may contain continuous fluid, with the level represented by a non-negative real number $X_{j} \in \mathbb{R}_{\geq 0}\left(1 \leq j \leq\left|P c_{N}\right|\right)$. Then the complete hybrid (discrete-continuous) marking of $N$ is a pair $(M, X)$, where $M=\left(M_{1}, \ldots, M_{\left|P d_{N}\right|}\right)$ is a discrete marking and $X=\left(X_{1}, \ldots, X_{\left|P c_{N}\right|}\right)$ is a continuous marking. When needed, these vectors can also be seen as the mappings $M: P d_{N} \rightarrow \mathbb{N}$ with $M\left(p_{i}\right)=M_{i}\left(1 \leq i \leq\left|P d_{N}\right|\right)$ and $X: P c_{N} \rightarrow$ $\mathbb{R}_{\geq 0}$ with $X\left(q_{j}\right)=X_{j}\left(1 \leq j \leq\left|P c_{N}\right|\right)$. The set of all markings (reachability set) of $N$ is denoted by $R S(N)$. Then $D R S(N)=\{M \mid(M, X) \in R S(N)\}$ is the set of all discrete markings (discrete reachability set) of $N$. DRS $(N)$ will be formally defined later. Further, $C R S(N)=\{X \mid(M, X) \in R S(N)\} \subseteq$ $\mathbb{R}_{>0}^{\left|P c_{N}\right|}$ is the set of all continuous markings (continuous reachability set) of $N$. Every marking $(M, X) \in R S(N)$ evolves in time, hence, we can interpret it as a stochastic process $\{(M(\delta), X(\delta)) \mid \delta \geq 0\}$. Then the initial marking of $N$ is that at the zero time moment, i.e. $\mathcal{M}_{N}=\left(M_{N}, \mathbf{0}\right)=(M(0), X(0))$, where $X(0)=\mathbf{0}$ means that all the continuous places are initially empty.

Every transition $t \in T_{N}$ has a positive real instantaneous rate $\Omega_{N}(t, M) \in$ $\mathbb{R}_{>0}$ associated, which is a parameter of the exponential distribution governing the transition delay (being a random variable), when the current discrete marking is $M$. Transitions are labeled with actions, each representing a sort of activity that they model.

Every discrete arc $d a=(p, t)$ or $d a=(t, p)$, where $p \in P d_{N}$ and $t \in$ $T_{N}$, connects discrete places and transitions. It has a non-negative integervalued weight $W_{N}(d a) \in \mathbb{N}$ assigned, representing its multiplicity. The zero weight indicates that the corresponding discrete arc does not exist, since its multiplicity is zero in this case. In the discrete marking $M \in D R S(N)$, every continuous arc $c a=(q, t)$ or $c a=(t, q)$, where $q \in P c_{N}$ and $t \in T_{N}$, connects continuous places and transitions. It has a non-negative real-valued 
flow rate $R_{N}(c a, M) \in \mathbb{R}_{\geq 0}$ of fluid through $c a$, when the current discrete marking is $M$. The zero flow rate indicates that the fluid flow along the corresponding continuous arc is stopped in some discrete marking.

The graphical representation of LFSPNs resembles that for standard labeled Petri nets, but supplemented with the rates or weights, written near the corresponding transitions or arcs. Discrete places are drawn with ordinary circles while double concentric circles correspond to the continuous ones. Square boxes with the action names inside depict transitions and their labels. Discrete arcs are drawn as thin lines with arrows at the end while continuous arcs should represent pipes, so the latter are depicted by thick arrowed lines. If the rates or the weights are not given in the picture then they are assumed to be of no importance in the corresponding examples. The names of places and transitions are depicted near them when needed.

We now consider the behavior of LFSPNs.

Let $N$ be an LFSPN and $M$ be a discrete marking of $N$. A transition $t \in T_{N}$ is enabled in $M$ if $\forall p \in P d_{N} W_{N}(p, t) \leq M(p)$. Let Ena $(M)$ be the set of all transitions enabled in $M$. Transition firings are atomic operations, and only single transitions are fired at once. The enabling condition depends just on the discrete part of $N$ and it is like that for CTSPNs. Firing of a transition $t \in \operatorname{Ena}(M)$ changes $M$ to a discrete marking $\widetilde{M}$, such as $\forall p \in P d_{N} \widetilde{M}(p)=M(p)-W_{N}(p, t)+W_{N}(t, p)$ denoted by $M \stackrel{t}{\rightarrow}{ }_{\lambda} \widetilde{M}$, where $\lambda=\Omega_{N}(t, M)$. We write $M \stackrel{t}{\rightarrow} \widetilde{M}$ if $\exists \lambda M \stackrel{t}{\rightarrow}{ }_{\lambda} \widetilde{M}$ and $M \rightarrow \widetilde{M}$ if $\exists t M \stackrel{t}{\rightarrow} \widetilde{M}$. Let us define the discrete reachability set and graph of $N$.

Definition 2. Let $N$ be an LFSPN. The discrete reachability set of $N$, denoted by $D R S(N)$, is the minimal set of discrete markings such that $M_{N} \in D R S(N)$ or, if $M \in D R S(N)$ and $M \rightarrow \widetilde{M}$, then $\widetilde{M} \in D R S(N)$.

Definition 3. Let $N$ be an LFSPN. The discrete reachability graph of $N$ is a labeled transition system $\operatorname{DRG}(N)=\left(S_{N}, \mathcal{L}_{N}, \mathcal{T}_{N}, s_{N}\right)$, where

- the set of states is $S_{N}=D R S(N)$;

- the set of labels is $\mathcal{L}_{N}=T_{N} \times \mathbb{R}_{>0}$;

- the set of transitions is $\mathcal{T}_{N}=\left\{(M,(t, \lambda), \widetilde{M}) \mid M, \widetilde{M} \in D R S(N), M \stackrel{t}{\rightarrow}{ }_{\lambda} \widetilde{M}\right\} ;$

- the initial state is $s_{N}=M_{N}$.

\section{Discrete part of LFSPNs}

We have restricted the class of FSPNs underlying LFSPNs to those whose discrete part are CTSPNs, since the performance analysis of standard FSPNs with GSPNs as the discrete part is finally based on the CTMCs which are 
extracted from the underlying semi-Markov chains (SMCs) of GSPNs by removing vanishing states. Let us now consider the behavior of the discrete part of LFSPNs, which is a labeled CTSPN.

For an LFSPN $N$, a continuous random variable $\xi(M)$ is associated with every discrete marking $M \in D R S(N)$. The variable captures a residence (sojourn) time in $M$. We implement the race semantics, in which the fastest stochastic transition (i.e. that with the minimal exponentially distributed firing delay) fires first. Hence, the probability distribution function (PDF) of the sojourn time in $M$ is that of the minimal firing delay of transitions from $\operatorname{Ena}(M)$. Since exponential distributions are closed under minimum, the sojourn time in $M$ is (again) exponentially distributed with a parameter that is called the exit rate from the discrete marking $M$, defined as

$$
R E(M)=\sum_{t \in \operatorname{Ena}(M)} \Omega_{N}(t, M) .
$$

Hence, the PDF of the sojourn time in $M$ (the probability of the residence time in $M$ being less than $\delta)$ is $F_{\xi(M)}(\delta)=\mathrm{P}(\xi(M)<\delta)=1-$ $e^{-R E(M) \delta}(\delta \geq 0)$. Then the probability density function of the residence time in $M$ (the limit probability of staying in $M$ at the time $\delta$ ) is $f_{\xi(M)}(\delta)=$ $\lim _{\Delta \rightarrow 0} \frac{F_{\xi(M)}(\delta+\Delta)-F_{\xi(M)}(\delta)}{\Delta}=\frac{d F_{\xi(M)}(\delta)}{d \delta}=R E(M) e^{-R E(M) \delta}(\delta \geq 0)$. The mean value (average, expectation) formula for the exponential distribution allows us to calculate the average sojourn time in $M$ as $\mathrm{M}(\xi(M))=$ $\int_{0}^{\infty} \delta f_{\xi(M)}(\delta) d \delta=\frac{1}{R E(M)}$.

The average sojourn time in the discrete marking $M$ is

$$
S J(M)=\frac{1}{\sum_{t \in \operatorname{Ena}(M)} \Omega_{N}(t, M)} .
$$

The average sojourn time vector of $N$, denoted by $S J$, has the elements $S J(M), M \in D R S(N)$.

To evaluate performance with the use of the discrete part of $N$, we should investigate the stochastic process associated with it. The process is the underlying continuous time Markov chain, denoted by $C T M C(N)$.

Let $M, \widetilde{M} \in D R S(N)$. The rate of moving from $M$ to $\widetilde{M}$ by firing any transition is

$$
R M(M, \widetilde{M})=\sum_{\{t \mid M \stackrel{t}{\rightarrow} \widetilde{M}\}} \Omega_{N}(t, M) .
$$

Definition 4. Let $N$ be an LFSPN. The underlying continuous time Markov chain $(C T M C)$ of $N$, denoted by $C T M C(N)$, has the state space $\operatorname{DRS}(N)$, the initial state $M_{N}$ and the transitions $M \rightarrow_{\lambda} \widetilde{M}$ if $M \rightarrow \widetilde{M}$, where $\lambda=R M(M, \widetilde{M})$.

Let $N$ be an LFSPN. The elements $\mathcal{Q}_{i j}(1 \leq i, j \leq n=|D R S(N)|)$ of the transition rate matrix (TRM) $\mathbf{Q}$ for $C T M C(N)$ are defined as 


$$
\mathcal{Q}_{i j}= \begin{cases}R M\left(M_{i}, M_{j}\right), & \text { if } i \neq j ; \\ -\sum_{\{k \mid 1 \leq k \leq n, k \neq i\}} R M\left(M_{i}, M_{k}\right), & \text { if } i=j .\end{cases}
$$

The transient probability mass function $(\mathrm{PMF}) \varphi(\delta)=\left(\varphi_{1}(\delta), \ldots, \varphi_{n}(\delta)\right)$ for $C T M C(N)$ is calculated via matrix exponent as

$$
\varphi(\delta)=\varphi(0) e^{\mathbf{Q} \delta},
$$

where $\varphi(0)=\left(\varphi_{1}(0), \ldots, \varphi_{n}(0)\right)$ is the initial $\operatorname{PMF} \varphi_{i}(0)= \begin{cases}1, & \text { if } M_{i}=M_{N} \\ 0, & \text { otherwise. }\end{cases}$

The steady-state $\operatorname{PMF} \varphi=\left(\varphi_{1}, \ldots, \varphi_{n}\right)$ for $C T M C(N)$ is a solution of the linear equation system

$$
\left\{\begin{array}{l}
\varphi \mathbf{Q}=\mathbf{0} \\
\varphi \mathbf{1}^{T}=1
\end{array},\right.
$$

where $\mathbf{0}$ is a row vector of $n$ values 0 and $\mathbf{1}$ is that of $n$ values 1 .

Note that the vector $\varphi$ exists and is unique, if $C T M C(N)$ is ergodic and we have $\varphi=\lim _{\delta \rightarrow \infty} \varphi(\delta)$.

Let $N$ be an LFSPN. The following steady-state discrete performance indices (measures) can be calculated based on the steady-state $\operatorname{PMF} \varphi$ for $C T M C(N)[22,8,5,23,2]$.

- The time fraction spent in the set of discrete markings $S \subseteq D R S(N)$ is

$$
\operatorname{TimeFract}(S)=\sum_{\left\{i \mid M_{i} \in S\right\}} \varphi_{i} \text {. }
$$

- The probability that $k \geq 0$ tokens are contained in a discrete place $p$ is

$$
\text { Tokens }(p, k)=\sum_{\left\{i \mid M_{i}(p)=k, M_{i} \in D R S(N)\right\}} \varphi_{i} .
$$

Then the PMF of the number of tokens in $p$ is

Tokens $(p)=($ Tokens $(p, 0)$, Tokens $(p, 1), \ldots)$.

- The probability of event $\mathcal{A}$ defined through the set of discrete markings $D R S_{\mathcal{A}}(N) \subseteq D R S(N)$ is

$$
\operatorname{Prob}(\mathcal{A})=\sum_{\left\{i \mid M_{i} \in D R S_{\mathcal{A}}(N)\right\}} \varphi_{i} .
$$

- The average number of tokens in a discrete place $p$ is

$$
\operatorname{TokensNum}(p)=\sum_{k \geq 1} \operatorname{Tokens}(p, k) \cdot k=\sum_{\left\{i \mid M_{i}(p) \geq 1, M_{i} \in D R S(N)\right\}} \varphi_{i} M_{i}(p) .
$$

- The firing frequency (throughput) of a transition $t \in T_{N}$ (average number of firings per unit of time) is

$$
\text { FiringFreq }(t)=\sum_{\left\{i \mid t \in \operatorname{Ena}\left(M_{i}\right), M_{i} \in D R S(N)\right\}} \varphi_{i} \Omega_{N}\left(t, M_{i}\right) .
$$

- The exit/entrance frequency of a discrete marking $M_{i} \in D R S(N)$ $(1 \leq i \leq n)$ (average number of exits/entrances per unit of time) is 


$$
\operatorname{ExitFreq}\left(M_{i}\right)=\varphi_{i} R E\left(M_{i}\right)=\frac{\varphi_{i}}{S J\left(M_{i}\right)} .
$$

- The probability of the event determined by a reward function $r\left(M_{i}\right)=$ $r_{i}\left(0 \leq r_{i} \leq 1,1 \leq i \leq n\right)$ of the discrete markings is

$$
\operatorname{Prob}(r)=\sum_{\left\{i \mid M_{i} \in D R S(N)\right\}} \varphi_{i} r_{i} .
$$

- The traversal frequency of the move from a discrete marking $M_{i}$ to a discrete marking $M_{j} \in D R S(N)(1 \leq i, j \leq n)$ (average number of traversals per unit of time) is

$$
\operatorname{TravFreq}\left(M_{i}, M_{j}\right)=\varphi_{i} R M\left(M_{i}, M_{j}\right) .
$$

\section{Continuous part of LFSPNs}

Consider the impact the discrete part of LFSPNs has on their continuous part, which is a stochastic fluid model (SFM). We investigate LFSPNs with a single continuous place, since the definitions and subsequent results on the fluid bisimulation can be transferred straightforwardly to the case of several continuous places, where multidimensional SFMs have to be explored.

Let $N$ be an LFSPN such that $P c_{N}=\{q\}$ and $M(\delta) \in D R S(N)$ be its discrete marking at the time $\delta \geq 0$. Every continuous arc $c a=(q, t)$ or $c a=(t, q)$, where $t \in T_{N}$, changes the fluid level in the continuous place $q$ at the time $\delta$ with the flow rate $R_{N}(c a, M(\delta))$. This means that in the discrete marking $M(\delta)$ fluid can leave $q$ along the continuous arc $(q, t)$ with the rate $R_{N}((q, t), M(\delta))$ and can enter $q$ along the continuous arc $(t, q)$ with the rate $R_{N}((t, q), M(\delta))$ for every transition $t \in \operatorname{Ena}(M(\delta))$.

The potential rate of the fluid level change (fluid flow rate) for the continuous place $q$ in the discrete marking $M(\delta)$ is

$$
R P(M(\delta))=\sum_{t \in \operatorname{Ena}(M(\delta))} R_{N}((t, q), M(\delta))-R_{N}((q, t), M(\delta)) .
$$

Let $X(\delta)$ be the fluid level in $q$ at the time $\delta$. It is clear that the fluid level in a continuous place can never be negative. Therefore, $X(\delta)$ satisfies the following ordinary differential equation describing the actual fluid flow rate for the continuous place $q$ in the marking $(M(\delta), X(\delta))$ :

$R A(M(\delta), X(\delta))=\frac{d X(\delta)}{d \delta}= \begin{cases}\max \{R P(M(\delta)), 0\}, & \text { if } X(\delta)=0 ; \\ R P(M(\delta)), & \text { if }(X(\delta)>0) \wedge \\ 0, & \left(R P\left(M\left(\delta^{-}\right)\right) R P\left(M\left(\delta^{+}\right)\right) \geq 0\right) \\ & \left(R P\left(M\left(\delta^{-}\right)\right) R P\left(M\left(\delta^{+}\right)\right)<0\right) .\end{cases}$

In the first case considered in the definition above, we have $X(\delta)=$ 0 . In this case, if $R P(M(\delta)) \geq 0$ then the fluid level is growing and the derivative is equal to the potential rate. Otherwise, if $R P(M(\delta))<0$ then 
we should prevent the fluid level from crossing the lower boundary (zero) by stopping the fluid flow. For an explanation of the more complex second and third cases please refer to $[20,11,15]$. Note that $\frac{d X(\delta)}{d \delta}$ is a piecewise constant function of $X(\delta)$; hence, for each different "constant" segment we have $\frac{d X(\delta)}{d \delta}=R P(M(\delta))$ or $\frac{d X(\delta)}{d \delta}=0$ and, therefore, we can suppose that within each such segment $R P(M(\delta))$ or 0 are the actual fluid flow rates for the continuous place $q$ in the marking $(M(\delta), X(\delta))$. While constructing differential equations that describe the behavior of SFMs associated with LFSPNs, we are interested only in the segments where $\frac{d X(\delta)}{d \delta}=R P(M(\delta))$. The SFMs behavior within the remaining segments, where $\frac{d X(\delta)}{d \delta}=0$, is completely comprised by the buffer empty probability function that collects the probability mass at the lower boundary.

The elements $\mathcal{R}_{i j}(1 \leq i, j \leq n=|D R S(N)|)$ of the fluid rate matrix (FRM) $\mathbf{R}$ for the continuous place $q$ are defined as

$$
\mathcal{R}_{i j}= \begin{cases}R P\left(M_{i}\right), & \text { if } i=j ; \\ 0, & \text { if } i \neq j .\end{cases}
$$

The underlying SFM of LFSPNs is the first order, infinite buffer, homogeneous Markov fluid model $[11,15]$. For an LFSPN $N$, the discrete part of its SFM is the CTMC CTMC $(N)$ with the TRM Q. The evolution of the continuous part of the SFM (fluid flow drift) is described by the FRM R.

Consider the stationary behavior of the SFM associated with an LFSPN $N$. We do not discuss here in detail the conditions under which the steady state for the underlying SFM exists and is unique, since this topic has been extensively explored in $[20,11,15]$. Particularly, according to $[20,15]$, the steady-state PDF exists (the transient functions approach their stationary values, as the time parameter $\delta$ tends to infinity in the transient equations) when the underlying SFM is a Markov fluid model whose fluid flow drift (described by the matrix $\mathbf{R}$ ) and transition rates (described by the matrix Q) are fluid level independent and the following stability condition holds:

$$
\operatorname{FluidFlow}(q)=\sum_{i=1}^{n} \varphi_{i} R P\left(M_{i}\right)=\varphi \mathbf{R} \mathbf{1}^{T}<0,
$$

stating that the steady-state mean potential fluid flow rate for the continuous place $q$ is negative. Stable infinite buffer models usually converge, hence, the existing steady-state PDF is also unique in this case.

We introduce the following steady-state probability functions, obtained from the transient ones by taking the limit $\delta \rightarrow \infty$.

- $\varphi_{i}=\lim _{\delta \rightarrow \infty} \mathrm{P}\left(M(\delta)=M_{i}\right)$ is the steady-state discrete marking probability;

- $\ell_{i}=\lim _{\delta \rightarrow \infty} \mathrm{P}\left(X(\delta)=0, M(\delta)=M_{i}\right)$ is the steady-state buffer empty probability (probability mass at the lower boundary); 
- $F_{i}(x)=\lim _{\delta \rightarrow \infty} \mathrm{P}\left(X(\delta)<x, M(\delta)=M_{i}\right)$ is the steady-state fluid probability distribution function;

- $f_{i}(x)=\frac{d F_{i}(x)}{d x}=\lim _{\delta \rightarrow \infty} \lim _{h \rightarrow 0} \frac{\mathrm{P}\left(x<X(\delta)<x+h, M(\delta)=M_{i}\right)}{h}$ is the steadystate fluid probability density function.

Let $\varphi, \ell, F(x), f(x)$ be the row vectors with the elements $\varphi_{i}, \ell_{i}, F_{i}(x)$, $f_{i}(x)$, respectively $(1 \leq i \leq n)$.

By the total probability law for the stationary behavior, we have

$$
\ell+\int_{0}^{\infty} f(x) d x=\varphi .
$$

The ordinary differential equations describing the stationary behavior are

$$
\frac{d F(x)}{d x} \mathbf{R}=F(x) \mathbf{Q}, x>0 ; \quad \frac{d f(x)}{d x} \mathbf{R}=f(x) \mathbf{Q}, x>0 .
$$

Note that we have $\frac{d F(x)}{d x}=f(x), F(0)=\ell, F(\infty)=\varphi$.

The ordinary differential equation for the steady-state buffer empty probabilities (stationary lower boundary conditions) are

$$
f(0) \mathbf{R}=\ell \mathbf{Q} .
$$

The stationary lower boundary constraint is: if $\mathcal{R}_{i i}=R P\left(M_{i}\right)>0$ then $F_{i}(0)=\ell_{i}=0(1 \leq i \leq n)$.

The stationary normalizing condition is

$$
\ell \mathbf{1}^{T}+\int_{0}^{\infty} f(x) d x \mathbf{1}^{T}=1
$$

where 1 is a row vector of $n$ values 1 .

The solutions of the equations for $F(x)$ and $f(x)$ in the form of $m a-$ trix exponent are $F(x)=\ell e^{x \mathbf{Q R} \mathbf{R}^{-1}}$ and $f(x)=\ell \mathbf{Q} \mathbf{R}^{-1} e^{x \mathbf{Q R}^{-1}}$, respectively. Since the steady-state existence implies boundedness of the SFM associated with an LFSPN and we do not have a finite upper fluid level bound, the positive eigenvalues of $\mathbf{Q} \mathbf{R}^{-1}$ must be excluded. Moreover, $\mathbf{R}^{-1}$ does not exist if for some $i(1 \leq i \leq n)$ we have $\mathcal{R}_{i i}=0$. These difficulties are avoided in the alternative solution method for $F(x)$ called spectral decomposition $[26,20,11,15,12]$, which we outline below.

Let us define the sets of negative discrete markings of $N$ as $D R S^{-}(N)=$ $\{M \in D R S(N) \mid R P(M)<0\}$, zero discrete markings of $N$ as $D R S^{0}(N)=$ $\{M \in D R S(N) \mid R P(M)=0\}$ and positive discrete markings of $N$ as $D R S^{+}(N)=\{M \in D R S(N) \mid R P(M)>0\}$. The spectral decomposition is $F(x)=\sum_{j=1}^{m} a_{j} e^{\gamma_{j} x} v_{j}$, where $a_{j}$ are some scalar coefficients, $\gamma_{j}$ are the eigenvalues and $v_{j}=\left(v_{j 1}, \ldots, v_{j n}\right)$ are the eigenvectors of $\mathbf{Q R} \mathbf{R}^{-1}$. Thus, each $v_{j}$ is the solution of the equation $v_{j}\left(\mathbf{Q R}^{-1}-\gamma_{j} \mathbf{I}\right)=0$, where $\mathbf{I}$ is the identity matrix of the order $n$, hence, it holds $v_{j}\left(\mathbf{Q}-\gamma_{j} \mathbf{R}\right)=0$.

Since for each non-zero $v_{j}$ we must have $\left|\mathbf{Q}-\gamma_{j} \mathbf{R}\right|=0$, the number of solutions $\gamma_{1}, \ldots, \gamma_{m}$ is the number of non-zero elements among $\mathcal{R}_{i i}=$ $R P\left(M_{i}\right) \quad(1 \leq i \leq n)$, i.e. $m=\left|D R S^{-}(N)\right|+\left|D R S^{+}(N)\right|$. We have 1 zero eigenvalue, $\left|D R S^{+}(N)\right|$ eigenvalues with a negative real part and 
$\left|D R S^{-}(N)\right|-1$ eigenvalues with a positive real part. Let us reorder all the eigenvalues according to the sign of their real part (first, with a zero real part; then with a negative one; at last, with a positive one). The boundedness of $F(x)$ requires $a_{j}=0$ if $\operatorname{Re}\left(\gamma_{j}\right)>0(1 \leq j \leq m)$. Further, for the zero eigenvalue $\gamma_{1}=0$ we have $a_{1} e^{\gamma_{1} x} v_{1}=a_{1} v_{1}$, and for the corresponding eigenvector it holds $v_{1} \mathbf{Q}=0$. Then $F(x)=a_{1} v_{1}+\sum_{k=2}^{\left|D R S^{+}(N)\right|+1} a_{k} e^{\gamma_{k} x} v_{k}$, where $\operatorname{Re}\left(\gamma_{k}\right)<0\left(2 \leq k \leq\left|D R S^{+}(N)\right|+1\right)$. Remember that $\varphi=F(\infty)=a_{1} v_{1}$, hence, $F(x)=\varphi+\sum_{k=2}^{\left|D R S^{+}(N)\right|+1} a_{k} e^{\gamma_{k} x} v_{k}$.

It remains to find $\left|D R S^{+}(N)\right|$ coefficients $a_{k}$ corresponding to the eigenvalues $\gamma_{k}\left(2 \leq k \leq\left|D R S^{+}(N)\right|+1\right)$. Remember the stationary lower boundary constraint: if $\mathcal{R}_{l l}=R P\left(M_{l}\right)>0$ then $F_{l}(0)=\ell_{l}=0$. Then for each positive discrete marking $M_{l} \in D R S^{+}(N)$ we have $F_{l}(0)=\varphi_{l}+$ $\sum_{k=2}^{\left|D R S^{+}(N)\right|+1} a_{k} v_{k l}=0$. We obtain a system of $\left|D R S^{+}(N)\right|$ independent linear equations with $\left|D R S^{+}(N)\right|$ unknowns, for which a unique solution exists.

Then, using $F(x)$, we can find $f(x)=\frac{d F(x)}{d x}$ and $\ell=F(0)$.

Let $N$ be an LFSPN. The following steady-state continuous performance indices (measures) can be calculated based on the steady-state fluid probability density function $f(x)$ for the SFM of $N[13,14,11,21]$.

- The mean potential fluid flow rate for the continuous place $q$ is

$$
\operatorname{FluidFlow~}(q)=\sum_{\left\{i \mid M_{i} \in D R S(N)\right\}}\left(\ell_{i}+\int_{0}^{\infty} f_{i}(x) d x\right) R P\left(M_{i}\right)=
$$

- The probability of a positive fluid level in a continuous place $q$ is

$$
\begin{gathered}
\text { FluidLevel }(q)=\sum_{\left\{i \mid M_{i} \in D R S(N)\right\}}\left(\ell_{i} \cdot 0+\int_{0}^{\infty} f_{i}(x) \cdot 1 d x\right)= \\
\sum_{\left\{i \mid M_{i} \in D R S(N)\right\}} \int_{0}^{\infty} f_{i}(x) d x=\sum_{\left\{i \mid M_{i} \in D R S(N)\right\}}\left(\varphi_{i}-\ell_{i}\right) .
\end{gathered}
$$

- The probability of the event determined by a hybrid reward function $r\left(M_{i}, x\right)=r_{i}(x)\left(0 \leq r_{i}(x) \leq 1,1 \leq i \leq n\right)$ of the markings is

$$
\operatorname{Prob}(r)=\sum_{\left\{i \mid M_{i} \in D R S(N)\right\}}\left(\ell_{i} r_{i}(0)+\int_{0}^{\infty} f_{i}(x) r_{i}(x) d x\right) .
$$




\section{Fluid bisimulation equivalence}

Bisimulation equivalences respect particular points of choice in the behavior of a system. To define fluid bisimulation equivalence, we have to consider a bisimulation being an equivalence relation that partitions the states of the union of the discrete reachability graphs $D R G(N)$ and $D R G\left(N^{\prime}\right)$ of the LFSPNs $N$ and $N^{\prime}$. For $N$ and $N^{\prime}$ to be bisimulation equivalent, the initial states $M_{N}$ and $M_{N^{\prime}}$ of their discrete reachability graphs should be related by a bisimulation having the following transfer property: if two states are related then in each of them the same action can occur, leading with the identical overall rate from each of the two states to the same equivalence class for every such action.

The definition of fluid bisimulation must be given at the level of LFSPNs, but it must use the transition rates of the extracted CTMC. These rates cannot be easily (i.e. with a simple expression) defined at the level of more general LFSPNs, whose discrete part is labeled GSPNs. In addition, the labels of immediate transitions are lost and their individual probabilities are redistributed while GSPNs are transformed into CTSPNs. The individual probabilities of immediate transitions are "dissolved" in the total transition rates between tangible states when vanishing states are eliminated from SMCs while reducing them to CTMCs. Therefore, to make the definition of fluid bisimulation less intricate and complex, we have decided to consider only LFSPNs with labeled CTSPNs as their discrete part. Then the underlying stochastic process of the discrete part of LFSPNs will be that of CTSPNs, i.e. a CTMC.

The novelty of the fluid bisimulation definition with respect to that of the Markovian bisimulations from [6, 19,3] is that, for each pair of bisimilar discrete markings of $N$ and $N^{\prime}$, we require coincidence of the fluid flow rates of the corresponding (i.e. related by a correspondence bijection) continuous places of $N$ and $N^{\prime}$ in these two discrete markings.

We first propose some helpful extensions of the rate functions for the discrete marking changes and for the fluid flow in continuous places. Let $N$ be an LFSPN and $\mathcal{H} \subseteq D R S(N)$. Then, for each $M \in D R S(N)$ and $a \in$ Act, we write $M \stackrel{a}{\rightarrow}_{\lambda} \mathcal{H}$, where $\lambda=R M_{a}(M, \mathcal{H})$ is the overall rate to move from $M$ into the set of discrete markings $\mathcal{H}$ by action a, defined as

$$
R M_{a}(M, \mathcal{H})=\sum_{\{t \mid \exists \widetilde{M} \in \mathcal{H}}{ }_{\left.M \stackrel{t}{\rightarrow} \widetilde{M}, L_{N}(t)=a\right\}} \Omega_{N}(t, M) .
$$

We write $M \stackrel{a}{\rightarrow} \mathcal{H}$ if $\exists \lambda M \stackrel{a}{\rightarrow} \lambda \mathcal{H}$. Further, we write $M \rightarrow_{\lambda} \mathcal{H}$ if $\exists a M \stackrel{a}{\rightarrow} \mathcal{H}$, where $\lambda=R M(M, \mathcal{H})$ is the overall rate to move from $M$ into the set of discrete markings $\mathcal{H}$ by any actions, defined as

$$
R M(M, \mathcal{H})=\sum_{\{t \mid \exists \widetilde{M} \in \mathcal{H} M \stackrel{t}{\rightarrow} \widetilde{M}\}} \Omega_{N}(t, M) .
$$


To construct a fluid bisimulation between LFSPNs $N$ and $N^{\prime}$, we should consider the "composite" set of their discrete markings $D R S(N) \cup D R S\left(N^{\prime}\right)$ since we have to identify the rates to come from any two equivalent discrete markings into the same "composite" equivalence class (with respect to the fluid bisimulation). Note that, for $N \neq N^{\prime}$, transitions starting from the discrete markings of $D R S(N)$ (or $D R S\left(N^{\prime}\right)$ ) always lead to those from the same set since $D R S(N) \cap D R S\left(N^{\prime}\right)=\emptyset$, and this allows us to "mix" the sets of discrete markings in the definition of fluid bisimulation.

Let $P c_{N}=\{q\}$ and $P c_{N^{\prime}}=\left\{q^{\prime}\right\}$. In this case, the continuous place $q^{\prime}$ of $N$ corresponds to $q$ of $N$, according to a trivial correspondence bijection $\beta: P c_{N} \rightarrow P c_{N^{\prime}}$ such that $\beta(q)=q^{\prime}$. Then for $M \in D R S(N)$ (or $M^{\prime} \in D R S\left(N^{\prime}\right)$ ) we denote by $R P(M)$ the fluid level change rate for the continuous place $q$ (or for the corresponding one $q^{\prime}$ ), i.e. the argument discrete marking determines for which of the two continuous places, $q$ or $q^{\prime}$, the flow rate function $R P$ is taken. Note that if $N$ and $N^{\prime}$ have more than one continuous place and there exists a correspondence bijection $\beta: P c_{N} \rightarrow P c_{N^{\prime}}$, then we should consider several flow rate functions $R P_{i}\left(1 \leq i \leq\left|P c_{N}\right|=\left|P c_{N^{\prime}}\right|\right)$ in the same manner, i.e. each $R P_{i}$ is used for the pair of the corresponding continuous places $q_{i} \in P c_{N}$ and $\beta\left(q_{i}\right)=q_{i}^{\prime} \in P c_{N^{\prime}}$.

Definition 5. Let $N$ and $N^{\prime}$ be LFSPNs with $P c_{N}=\{q\}, P c_{N^{\prime}}=\left\{q^{\prime}\right\}$, and $q^{\prime}$ corresponds to $q$. An equivalence relation $\mathcal{R} \subseteq\left(D R S(N) \cup D R S\left(N^{\prime}\right)\right)^{2}$

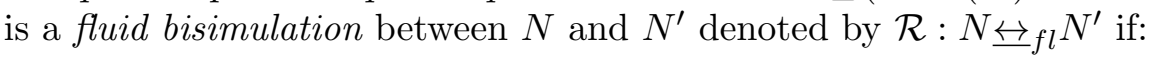

1. $\left(M_{N}, M_{N^{\prime}}\right) \in \mathcal{R}$.

2. $\left(M_{1}, M_{2}\right) \in \mathcal{R} \Rightarrow R P\left(M_{1}\right)=R P\left(M_{2}\right), \forall \mathcal{H} \in\left(D R S(N) \cup D R S\left(N^{\prime}\right)\right) / \mathcal{R}$ $\forall a \in$ Act $M_{1} \stackrel{a}{\rightarrow}_{\lambda} \mathcal{H} \Leftrightarrow M_{2} \stackrel{a}{\rightarrow}_{\lambda} \mathcal{H}$.

Two LFSPNs $N$ and $N^{\prime}$ are fluid bisimulation equivalent, denoted by

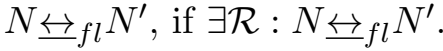

Let $\mathcal{R}_{f l}\left(N, N^{\prime}\right)=\bigcup\left\{\mathcal{R} \mid \mathcal{R}: N \biguplus_{f l} N^{\prime}\right\}$ be the union of all fluid bisimulations between $N$ and $N^{\prime}$. The following proposition proves that $\mathcal{R}_{f l}\left(N, N^{\prime}\right)$

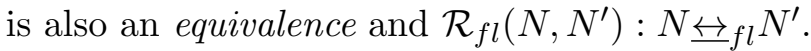

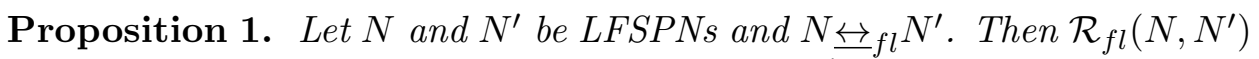
is the largest fluid bisimulation between $N$ and $N^{\prime}$.

Proof. Analogous to that of Proposition 8.2.1 from [19], which establishes the result for strong equivalence.

Let $N, N^{\prime}$ be LFSPNs with $\mathcal{R}: N \biguplus_{f l} N^{\prime}$ and $\mathcal{H} \in\left(D R S(N) \cup D R S\left(N^{\prime}\right)\right) / \mathcal{R}$. We now present a number of important equalities and helpful notations based on the rate functions $R M_{a}, R M, R P$ and probability function $S J$. 
1. We have $\forall M_{1}, M_{2} \in \mathcal{H} \forall \widetilde{\mathcal{H}} \in\left(D R S(N) \cup D R S\left(N^{\prime}\right)\right) /_{\mathcal{R}} \forall a \in A c t M_{1} \stackrel{a}{\rightarrow}{ }_{\lambda}$ $\widetilde{\mathcal{H}} \Leftrightarrow M_{2} \stackrel{a}{\rightarrow}_{\lambda} \widetilde{\mathcal{H}}$. Since the equality above is valid for all $M_{1}, M_{2} \in \mathcal{H}$, we can rewrite it as $\mathcal{H} \stackrel{a}{\rightarrow}_{\lambda} \widetilde{\mathcal{H}}$, where $\lambda=R M_{a}(\mathcal{H}, \widetilde{\mathcal{H}})=R M_{a}\left(M_{1}, \widetilde{\mathcal{H}}\right)=$ $R M_{a}\left(M_{2}, \widetilde{\mathcal{H}}\right)=R M_{a}(\mathcal{H} \cap D R S(N), \widetilde{\mathcal{H}})=R M_{a}\left(\mathcal{H} \cap D R S\left(N^{\prime}\right), \widetilde{\mathcal{H}}\right)$. Then we write $\mathcal{H} \stackrel{a}{\rightarrow} \widetilde{\mathcal{H}}$ if $\exists \lambda \mathcal{H} \stackrel{a}{\rightarrow}{ }_{\lambda} \widetilde{\mathcal{H}}$ and $\mathcal{H} \rightarrow \widetilde{\mathcal{H}}$ if $\exists a \mathcal{H} \stackrel{a}{\rightarrow} \widetilde{\mathcal{H}}$.

Since the transitions from the discrete markings of $D R S(N)$ always lead to those from the same set, we have $\forall M \in D R S(N) \forall a \in$ Act $R M_{a}(M, \widetilde{\mathcal{H}})=R M_{a}(M, \widetilde{\mathcal{H}} \cap D R S(N))$. Hence, $\forall M \in \mathcal{H} \cap D R S(N)$ $\forall a \in \operatorname{Act} R M_{a}(\mathcal{H}, \widetilde{\mathcal{H}})=R M_{a}(M, \widetilde{\mathcal{H}})=R M_{a}(M, \widetilde{\mathcal{H}} \cap D R S(N))=$ $R M_{a}(\mathcal{H} \cap D R S(N), \widetilde{\mathcal{H}} \cap D R S(N))$. The same is true for $D R S\left(N^{\prime}\right)$. Thus, $\forall \widetilde{\mathcal{H}} \in\left(D R S(N) \cup D R S\left(N^{\prime}\right)\right) / \mathcal{R}$

$$
\begin{gathered}
R M_{a}(\mathcal{H} \cap D R S(N), \widetilde{\mathcal{H}} \cap D R S(N))=R M_{a}(\mathcal{H}, \widetilde{\mathcal{H}})= \\
R M_{a}\left(\mathcal{H} \cap D R S\left(N^{\prime}\right), \widetilde{\mathcal{H}} \cap D R S\left(N^{\prime}\right)\right) .
\end{gathered}
$$

2. We have $\forall M_{1}, M_{2} \in \mathcal{H} \forall \widetilde{\mathcal{H}} \in\left(D R S(N) \cup D R S\left(N^{\prime}\right)\right) / \mathcal{R} R M\left(M_{1}, \widetilde{\mathcal{H}}\right)=$ $\sum_{\left\{t \mid \exists \widetilde{M_{1}} \in \widetilde{\mathcal{H}} M_{1} \stackrel{t}{\rightarrow} \widetilde{M}_{1}\right\}} \Omega_{N}\left(t, M_{1}\right)=$

$\sum_{a \in A c t} \sum_{\left\{t \mid \exists \widetilde{M}_{1} \in \widetilde{\mathcal{H}} M_{1} \stackrel{t}{\rightarrow} \widetilde{M}_{1}, L_{N}(t)=a\right\}} \Omega_{N}\left(t, M_{1}\right)=\sum_{a \in A c t} R M_{a}\left(M_{1}, \widetilde{\mathcal{H}}\right)=$ $\sum_{a \in A c t} R M_{a}\left(M_{2}, \widetilde{\mathcal{H}}\right)=\sum_{a \in A c t} \sum_{\left\{t \mid \exists \widetilde{M}_{2} \in \widetilde{\mathcal{H}} M_{2} \stackrel{t}{\rightarrow} \widetilde{M}_{2}, L_{N}(t)=a\right\}} \Omega_{N}\left(t, M_{2}\right)=$ $\sum_{\left\{t \mid \exists \widetilde{M}_{2} \in \widetilde{\mathcal{H}} M_{2} \stackrel{t}{\rightarrow} \widetilde{M}_{2}\right\}} \Omega_{N}\left(t, M_{2}\right)=R M\left(M_{2}, \widetilde{\mathcal{H}}\right)$. Since the previous equality is valid for all $M_{1}, M_{2} \in \mathcal{H}$, we can denote $R M(\mathcal{H}, \widetilde{\mathcal{H}})=R M\left(M_{1}, \widetilde{\mathcal{H}}\right)=$ $R M\left(M_{2}, \widetilde{\mathcal{H}}\right)$. Then we write $\mathcal{H} \rightarrow_{\lambda} \widetilde{\mathcal{H}}$, where $\lambda=R M(\mathcal{H}, \widetilde{\mathcal{H}})=$ $R M\left(M_{1}, \widetilde{\mathcal{H}}\right)=R M\left(M_{2}, \widetilde{\mathcal{H}}\right)$.

Since the transitions from the discrete markings of $D R S(N)$ always lead to those from the same set, we have $\forall M \in D R S(N) R M(M, \widetilde{\mathcal{H}})=$ $R M(M, \widetilde{\mathcal{H}} \cap D R S(N))$. Hence, $\forall M \in \mathcal{H} \cap D R S(N) R M(\mathcal{H}, \widetilde{\mathcal{H}})=$ $R M(M, \widetilde{\mathcal{H}})=R M(M, \widetilde{\mathcal{H}} \cap D R S(N))=R M(\mathcal{H} \cap D R S(N), \widetilde{\mathcal{H}} \cap D R S(N))$. The same is true for $D R S\left(N^{\prime}\right)$. Thus, $\forall \widetilde{\mathcal{H}} \in\left(D R S(N) \cup D R S\left(N^{\prime}\right)\right) / \mathcal{R}$

$$
\begin{gathered}
R M(\mathcal{H} \cap D R S(N), \widetilde{\mathcal{H}} \cap D R S(N))=R M(\mathcal{H}, \widetilde{\mathcal{H}})= \\
R M\left(\mathcal{H} \cap D R S\left(N^{\prime}\right), \widetilde{\mathcal{H}} \cap D R S\left(N^{\prime}\right)\right) .
\end{gathered}
$$

3. We have $\forall M_{1}, M_{2} \in \mathcal{H} R P\left(M_{1}\right)=R P\left(M_{2}\right)$. Since the previous equality is valid for all $M_{1}, M_{2} \in \mathcal{H}$, we can denote $R P(\mathcal{H})=R P\left(M_{1}\right)=R P\left(M_{2}\right)$.

Since any argument discrete marking $M \in D R S(N) \cup D R S\left(N^{\prime}\right)$ completely determines for which continuous place the flow rate function $R P(M)$ is taken (either for $q$ if $M \in D R S(N)$ or for $q^{\prime}$ if $M \in$ $\left.D R S\left(N^{\prime}\right)\right)$, we have $\forall M \in \mathcal{H} \cap D R S(N) R P(\mathcal{H})=R P(M)=R P(\mathcal{H} \cap$ $D R S(N))$. The same is true for $D R S\left(N^{\prime}\right)$. Thus, 


$$
R P(\mathcal{H} \cap D R S(N))=R P(\mathcal{H})=R P\left(\mathcal{H} \cap D R S\left(N^{\prime}\right)\right) .
$$

4. We have $\forall M_{1}, M_{2} \in \mathcal{H} S J\left(M_{1}\right)=\frac{1}{\sum_{t \in \operatorname{Ena}\left(M_{1}\right)} \Omega_{N}\left(t, M_{1}\right)}=$

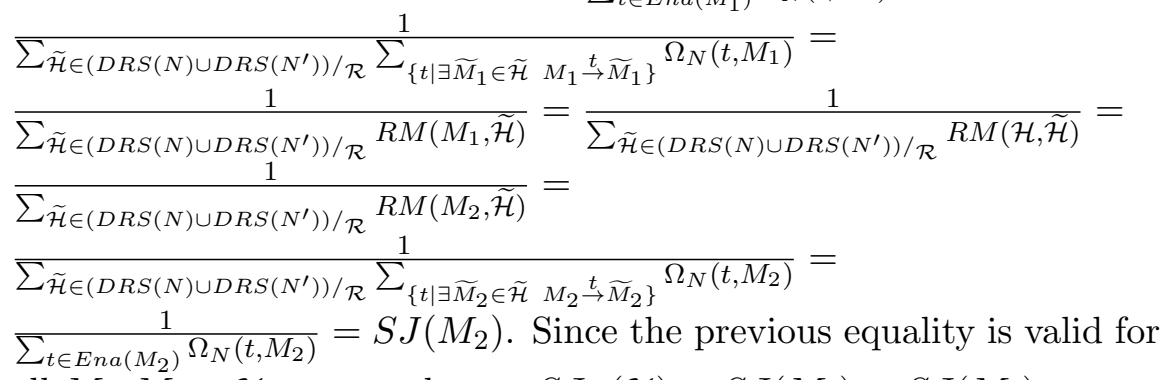
all $M_{1}, M_{2} \in \mathcal{H}$, we can denote $S J_{\mathcal{R}}(\mathcal{H})=S J\left(M_{1}\right)=S J\left(M_{2}\right)$.

Since any argument discrete marking $M \in D R S(N) \cup D R S\left(N^{\prime}\right)$ completely determines for which LFSPN the average sojourn time function $S J(M)$ is considered (either for $N$ if $M \in D R S(N)$, or for $N^{\prime}$ if $M \in D R S\left(N^{\prime}\right)$ ), we have $\forall M \in \mathcal{H} \cap D R S(N) S J(\mathcal{H})=S J(M)=$ $S J(\mathcal{H} \cap D R S(N))$. The same is true for $D R S\left(N^{\prime}\right)$. Thus,

$$
S J(\mathcal{H} \cap D R S(N))=S J(\mathcal{H})=S J\left(\mathcal{H} \cap D R S\left(N^{\prime}\right)\right) .
$$

\section{Preservation of the quantitative behavior}

It is clear that the proposed fluid bisimulation equivalence of LFSPNs preserves their qualitative (functional) behavior which is based on the actions assigned to the fired transitions. Let us examine if fluid bisimulation equivalence also preserves the quantitative (performance) behavior of LFSPNs, taken for the steady states of their underlying SFMs. The quantitative behavior takes into account the values of rates, probabilities and related functions, such as PDF.

The following proposition demonstrates that for two LFSPNs related by

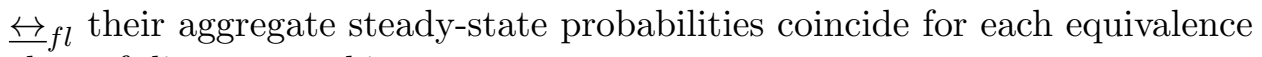
class of discrete markings.

Proposition 2. Let $N, N^{\prime}$ be LFSPNs with $\mathcal{R}: N_{f l}{ }_{f} N^{\prime}$ and $\varphi=\left(\varphi_{1}, \ldots, \varphi_{n}\right), n=|D R S(N)|$, be the steady-state PMF for CTMC $(N)$ and $\varphi^{\prime}=\left(\varphi_{1}^{\prime}, \ldots, \varphi_{m}^{\prime}\right), m=|D R S(N)|$, be the steady-state PMF for $C T M C\left(N^{\prime}\right)$. Then for all $\mathcal{H} \in\left(D R S(N) \cup D R S\left(N^{\prime}\right)\right) / \mathcal{R}$ we have

$$
\sum_{\left\{i \mid M_{i} \in \mathcal{H} \cap D R S(N)\right\}} \varphi_{i}=\sum_{\left\{j \mid M_{j}^{\prime} \in \mathcal{H} \cap D R S\left(N^{\prime}\right)\right\}} \varphi_{j}^{\prime} .
$$

Proof. The steady-state $\operatorname{PMF} \varphi=\left(\varphi_{1}, \ldots, \varphi_{n}\right)$ for $C T M C(N)$ is a solution of $\left\{\begin{array}{l}\varphi \mathbf{Q}=\mathbf{0} \\ \varphi \mathbf{1}^{T}=1\end{array}\right.$. Then for all $i(1 \leq i \leq n)$ we have $\left\{\begin{array}{l}\sum_{j=1}^{n} \mathcal{Q}_{j i} \varphi_{j}=0 \\ \sum_{j=1}^{n} \varphi_{j}=1\end{array}\right.$. By definition of $\mathcal{Q}_{i j}(1 \leq i, j \leq n)$ we have $\left\{\begin{array}{l}\sum_{j=1}^{n} R M\left(M_{j}, M_{i}\right) \varphi_{j}=0 \\ \sum_{j=1}^{n} \varphi_{j}=1\end{array}\right.$. 
Let $\mathcal{H} \in\left(D R S(N) \cup D R S\left(N^{\prime}\right)\right) / \mathcal{R}$. We sum the left and right sides of the first equation from the system above for all $i$ such that $M_{i} \in \mathcal{H} \cap D R S(N)$. The resulting equation is

$$
\sum_{\left\{i \mid M_{i} \in \mathcal{H} \cap D R S(N)\right\}} \sum_{j=1}^{n} R M\left(M_{j}, M_{i}\right) \varphi_{j}=0 .
$$

Let us denote the aggregate steady-state $\mathrm{PMF}$ for $C T M C(N)$ by $\varphi_{\mathcal{H} \cap D R S(N)}=\sum_{\left\{i \mid M_{i} \in \mathcal{H} \cap D R S(N)\right\}} \varphi_{i}$. Then, by the remark about $R M$, as a function of the equivalence classes (by fluid bisimulation), at the end of Section 5, for the left-hand side of the equation above, we come to $\sum_{\left\{i \mid M_{i} \in \mathcal{H} \cap D R S(N)\right\}} \sum_{j=1}^{n} R M\left(M_{j}, M_{i}\right) \varphi_{j}=$ $\sum_{j=1}^{n} \varphi_{j} \sum_{\left\{i \mid M_{i} \in \mathcal{H} \cap D R S(N)\right\}} R M\left(M_{j}, M_{i}\right)=\sum_{j=1}^{n} R M\left(M_{j}, \mathcal{H}\right) \varphi_{j}=$ $\sum_{\widetilde{\mathcal{H}} \in\left(D R S(N) \cup D R S\left(N^{\prime}\right)\right) / \mathcal{R}} \sum_{\left\{j \mid M_{j} \in \widetilde{\mathcal{H}} \cap D R S(N)\right\}} R M\left(M_{j}, \mathcal{H}\right) \varphi_{j}=$ $\sum_{\widetilde{\mathcal{H}} \in\left(D R S(N) \cup D R S\left(N^{\prime}\right)\right) / \mathcal{R}} \sum_{\left\{j \mid M_{j} \in \widetilde{\mathcal{H}} \cap D R S(N)\right\}} R M(\widetilde{\mathcal{H}}, \mathcal{H}) \varphi_{j}=$ $\sum_{\widetilde{\mathcal{H}} \in\left(D R S(N) \cup D R S\left(N^{\prime}\right)\right) / \mathcal{R}} R M(\widetilde{\mathcal{H}}, \mathcal{H}) \sum_{\left\{j \mid M_{j} \in \widetilde{\mathcal{H}} \cap D R S(N)\right\}} \varphi_{j}=$ $\sum_{\widetilde{\mathcal{H}} \in\left(D R S(N) \cup D R S\left(N^{\prime}\right)\right) / \mathcal{R}} R M(\widetilde{\mathcal{H}}, \mathcal{H}) \varphi_{\widetilde{\mathcal{H}} \cap D R S(N)}$.

For the left-hand side of the second equation from the system above, we have $\sum_{j=1}^{n} \varphi_{j}=\sum_{\tilde{\mathcal{H}} \in\left(D R S(N) \cup D R S\left(N^{\prime}\right)\right) / \mathcal{R}} \sum_{\left\{j \mid M_{j} \in \tilde{\mathcal{H}} \cap D R S(N)\right\}} \varphi_{j}=$ $\sum_{\tilde{\mathcal{H}} \in\left(D R S(N) \cup D R S\left(N^{\prime}\right)\right) / \mathcal{R}} \varphi_{\mathcal{H} \cap D R S(N)}$.

Thus, the aggregate linear equation system for $C T M C(N)$ is

$$
\left\{\begin{array}{l}
\sum_{\widetilde{\mathcal{H}} \in\left(D R S(N) \cup D R S\left(N^{\prime}\right)\right) / \mathcal{R}} R M(\widetilde{\mathcal{H}}, \mathcal{H}) \varphi_{\widetilde{\mathcal{H}} \cap D R S(N)}=0 \\
\sum_{\widetilde{\mathcal{H}} \in\left(D R S(N) \cup D R S\left(N^{\prime}\right)\right) / \mathcal{R}} \varphi_{\mathcal{H} \cap D R S(N)}=1
\end{array} .\right.
$$

Let us denote the aggregate steady-state PMF for $C T M C\left(N^{\prime}\right)$ by $\varphi_{\mathcal{H} \cap D R S\left(N^{\prime}\right)}^{\prime}=\sum_{\left\{j \mid M_{j}^{\prime} \in \mathcal{H} \cap D R S\left(N^{\prime}\right)\right\}} \varphi_{j}^{\prime}$. Then, in a similar way, the aggregate linear equation system for $C T M C\left(N^{\prime}\right)$ is

$$
\left\{\begin{array}{l}
\sum_{\widetilde{\mathcal{H}} \in\left(D R S(N) \cup D R S\left(N^{\prime}\right)\right) / \mathcal{R}} R M(\widetilde{\mathcal{H}}, \mathcal{H}) \varphi_{\widetilde{\mathcal{H}} \cap D R S\left(N^{\prime}\right)}^{\prime}=0 \\
\sum_{\widetilde{\mathcal{H}} \in\left(D R S(N) \cup D R S\left(N^{\prime}\right)\right) / \mathcal{R}} \varphi_{\mathcal{H} \cap D R S\left(N^{\prime}\right)}^{\prime}=1
\end{array} .\right.
$$

Let $\left(D R S(N) \cup D R S\left(N^{\prime}\right)\right) / \mathcal{R}=\left\{\mathcal{H}_{1}, \ldots, \mathcal{H}_{l}\right\}$. Then the aggregate steady-state PMFs $\varphi_{\mathcal{H}_{k} \cap D R S(N)}$ and $\varphi_{\mathcal{H}_{k} \cap D R S\left(N^{\prime}\right)}^{\prime}(1 \leq k \leq l)$ satisfy the same aggregate system of $l+1$ linear equations with $l$ independent equations and $l$ unknowns. The aggregate linear equation system has a unique solution when a single aggregate steady-state PMF exists, which is the case here. Hence, $\varphi_{\mathcal{H}_{k} \cap D R S(N)}=\varphi_{\mathcal{H}_{k} \cap D R S\left(N^{\prime}\right)}^{\prime}(1 \leq k \leq l)$.

The following proposition demonstrates that for two LFSPNs related by

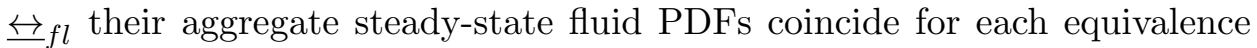
class of discrete markings.

Proposition 3. Let $N, N^{\prime}$ be LFSPNs with $\mathcal{R}: N \biguplus_{f l} N^{\prime}$ and $F(x)=$ $\left(F_{1}(x), \ldots, F_{n}(x)\right), n=|D R S(N)|$, be the steady-state fluid PDF for the 
$S F M$ of $N$ and $F^{\prime}(x)=\left(F_{1}^{\prime}(x), \ldots, F_{m}^{\prime}(x)\right), m=\left|D R S\left(N^{\prime}\right)\right|$, be the steadystate fluid PDF for the $S F M$ of $N^{\prime}$. Then for all $\mathcal{H} \in\left(D R S(N) \cup D R S\left(N^{\prime}\right)\right) / \mathcal{R}$ we have

$$
\sum_{\left\{i \mid M_{i} \in \mathcal{H} \cap D R S(N)\right\}} F_{i}(x)=\sum_{\left\{j \mid M_{j}^{\prime} \in \mathcal{H} \cap D R S\left(N^{\prime}\right)\right\}} F_{j}^{\prime}(x), x>0 .
$$

Proof. The ordinary differential equation characterizing the steady-state PDF for the SFM of $N$ is $\frac{d F(x)}{d x} \mathbf{R}=F(x) \mathbf{Q}, x>0$.

The upper boundary constraint is $F(\infty)=\varphi$, where $\varphi$ is the steady-state $\operatorname{PMF}$ for $C T M C(N)$.

Then for all $i(1 \leq i \leq n)$ we have

$$
\mathcal{R}_{i i} \frac{\bar{d} F_{i}(x)}{d x}=\sum_{j=1}^{n} \mathcal{Q}_{j i} F_{j}(x), x>0 .
$$

The upper boundary constraints are $\forall i(1 \leq i \leq n) F_{i}(\infty)=\varphi_{i}$, where $\varphi=\left(\varphi_{1}, \ldots, \varphi_{n}\right)$ is the steady-state PMF for $C T M C(N)$.

By definition of $\mathcal{R}_{i j}$ and $\mathcal{Q}_{i j}(1 \leq i, j \leq n)$ we have

$$
R P\left(M_{i}\right) \frac{d F_{i}(x)}{d x}=\sum_{j=1}^{n} R M\left(M_{j}, M_{i}\right) F_{j}(x), x>0 .
$$

Let $\mathcal{H} \in\left(D R S(N) \cup D R S\left(N^{\prime}\right)\right) / \mathcal{R}$. We sum the left and right sides of the equation above for all $i$ such that $M_{i} \in \mathcal{H} \cap D R S(N)$. The resulting equation is (where $x>0$ )

$$
\sum_{\left\{i \mid M_{i} \in \mathcal{H} \cap D R S(N)\right\}} R P\left(M_{i}\right) \frac{d F_{i}(x)}{d x}=\sum_{\left\{i \mid M_{i} \in \mathcal{H} \cap D R S(N)\right\}} \sum_{j=1}^{n} R M\left(M_{j}, M_{i}\right) F_{j}(x) .
$$

Let us denote the aggregate fluid flow PDF for the SFM of $N$ by $F_{\mathcal{H} \cap D R S(N)}(x)=\sum_{\left\{i \mid M_{i} \in \mathcal{H} \cap D R S(N)\right\}} F_{i}(x)$. By the remark about $R P$ (as a function of the equivalence classes) at the end of Section 5 , for the left-hand side of the equation above, we have $\sum_{\left\{i \mid M_{i} \in \mathcal{H} \cap D R S(N)\right\}} R P\left(M_{i}\right) \frac{d F_{i}(x)}{d x}=$ $\sum_{\left\{i \mid M_{i} \in \mathcal{H} \cap D R S(N)\right\}} R P(\mathcal{H}) \frac{d F_{i}(x)}{d x}=R P(\mathcal{H}) \sum_{\left\{i \mid M_{i} \in \mathcal{H} \cap D R S(N)\right\}} \frac{d F_{i}(x)}{d x}=$ $R P(\mathcal{H}) \frac{d}{d x}\left(\sum_{\left\{i \mid M_{i} \in \mathcal{H} \cap D R S(N)\right\}} F_{i}(x)\right)=R P(\mathcal{H}) \frac{d F_{\mathcal{H} \cap D R S(N)}(x)}{d x}$.

Analogously, for the right-hand side of the equation above, we have $\sum_{\left\{i \mid M_{i} \in \mathcal{H} \cap D R S(N)\right\}} \sum_{j=1}^{n} R M\left(M_{j}, M_{i}\right) F_{j}(x)=$ $\sum_{j=1}^{n} F_{j}(x) \sum_{\left\{i \mid M_{i} \in \mathcal{H} \cap D R S(N)\right\}} R M\left(M_{j}, M_{i}\right)=\sum_{j=1}^{n} R M\left(M_{j}, \mathcal{H}\right) F_{j}(x)=$ $\sum_{\widetilde{\mathcal{H}} \in\left(D R S(N) \cup D R S\left(N^{\prime}\right)\right) / \mathcal{R}} \sum_{\left\{j \mid M_{j} \in \widetilde{\mathcal{H}} \cap D R S(N)\right\}} R M\left(M_{j}, \mathcal{H}\right) F_{j}(x)=$ $\sum_{\widetilde{\mathcal{H}} \in\left(D R S(N) \cup D R S\left(N^{\prime}\right)\right) / \mathcal{R}} \sum_{\left\{j \mid M_{j} \in \widetilde{\mathcal{H}} \cap D R S(N)\right\}} R M(\widetilde{\mathcal{H}}, \mathcal{H}) F_{j}(x)=$ $\sum_{\tilde{\mathcal{H}} \in\left(D R S(N) \cup D R S\left(N^{\prime}\right)\right) /_{\mathcal{R}}} R M(\widetilde{\mathcal{H}}, \mathcal{H}) \sum_{\left\{j \mid M_{j} \in \widetilde{\mathcal{H}} \cap D R S(N)\right\}} F_{j}(x)=$ $\sum_{\widetilde{\mathcal{H}} \in\left(D R S(N) \cup D R S\left(N^{\prime}\right)\right) / \mathcal{R}} R M(\widetilde{\mathcal{H}}, \mathcal{H}) F_{\widetilde{\mathcal{H}} \cap D R S(N)}(x)$.

By combining both the resulting sides of the differential equation, we obtain the aggregate differential equation system for the SFM of $N$ (where $x>0)$ : 


$$
R P(\mathcal{H}) \frac{d F_{\mathcal{H} \cap D R S(N)}(x)}{d x}=\sum_{\widetilde{\mathcal{H}} \in\left(D R S(N) \cup D R S\left(N^{\prime}\right)\right) / \mathcal{R}} R M(\tilde{\mathcal{H}}, \mathcal{H}) F_{\widetilde{\mathcal{H}} \cap D R S(N)}(x)
$$

Let us denote the aggregate fluid flow PDF for the SFM of $N^{\prime}$ by

$F_{\mathcal{H} \cap D R S\left(N^{\prime}\right)}^{\prime}(x)=\sum_{\left\{j \mid M_{j}^{\prime} \in \mathcal{H} \cap D R S\left(N^{\prime}\right)\right\}} F_{j}^{\prime}(x)$. Then, in a similar way, we obtain the aggregate differential equation system for the SFM of $N^{\prime}$ (where $x>0)$ :

$$
R P(\mathcal{H}) \frac{d F_{\mathcal{H} \cap D R S\left(N^{\prime}\right)}^{\prime}(x)}{d x}=\sum_{\widetilde{\mathcal{H}} \in\left(D R S(N) \cup D R S\left(N^{\prime}\right)\right) / \mathcal{R}} R M(\widetilde{\mathcal{H}}, \mathcal{H}) F_{\widetilde{\mathcal{H}} \cap D R S\left(N^{\prime}\right)}^{\prime}(x) .
$$

By Proposition 2, the upper boundary constraints associated with the aggregate differential equation systems for the SFMs of $N$ and $N^{\prime}$ coincide: $F_{\mathcal{H} \cap D R S(N)}(\infty)=\sum_{\left\{i \mid M_{i} \in \mathcal{H} \cap D R S(N)\right\}} F_{i}(\infty)=\sum_{\left\{i \mid M_{i} \in \mathcal{H} \cap D R S(N)\right\}} \varphi_{i}=$ $\sum_{\left\{j \mid M_{j}^{\prime} \in \mathcal{H} \cap D R S\left(N^{\prime}\right)\right\}} \varphi_{i}^{\prime}=\sum_{\left\{j \mid M_{j}^{\prime} \in \mathcal{H} \cap D R S\left(N^{\prime}\right)\right\}} F_{j}^{\prime}(\infty)=F_{\mathcal{H} \cap D R S\left(N^{\prime}\right)}^{\prime}(\infty)$.

Let $\left(D R S(N) \cup D R S\left(N^{\prime}\right)\right) / \mathcal{R}=\left\{\mathcal{H}_{1}, \ldots, \mathcal{H}_{l}\right\}$. Similarly to the above results for $\mathcal{H} \in\left(D R S(N) \cup D R S\left(N^{\prime}\right)\right) / \mathcal{R}$, we can demonstrate that for each $\mathcal{H}_{k}(1 \leq k \leq l)$ the aggregate differential equation systems for the SFMs of $N$ and $N^{\prime}$ and the associated upper boundary constraints coincide.

For each $\mathcal{H}_{k}(1 \leq k \leq l)$, the lower boundary constraints are $\exists M_{i} \in \mathcal{H}_{k} \cap$ $D R S(N) R P\left(M_{i}\right)>0 \Rightarrow F_{i}(0)=0$ and $\exists M_{j}^{\prime} \in \mathcal{H}_{k} \cap D R S\left(N^{\prime}\right) R P\left(M_{j}^{\prime}\right)>$ $0 \Rightarrow F_{j}^{\prime}(0)=0$. Since $\forall M_{i} \in \mathcal{H}_{k} \cap D R S(N) \forall M_{j}^{\prime} \in \mathcal{H}_{k} \cap D R S\left(N^{\prime}\right) R P\left(M_{i}\right)=$ $R P\left(\mathcal{H}_{k} \cap D R S(N)\right)=R P\left(\mathcal{H}_{k}\right)=R P\left(\mathcal{H}_{k} \cap D R S\left(N^{\prime}\right)\right)=R P\left(M_{j}^{\prime}\right)$, we have $F_{\mathcal{H}_{k} \cap D R S(N)}(0)=0 \Leftarrow R P\left(\mathcal{H}_{k}\right)>0 \Rightarrow F_{\mathcal{H}_{k} \cap D R S\left(N^{\prime}\right)}^{\prime}(0)=0(1 \leq k \leq l)$.

Then the aggregate fluid flow PDFs $F_{\mathcal{H}_{k} \cap D R S(N)}(x)$ and $F_{\mathcal{H}_{k} \cap D R S\left(N^{\prime}\right)}^{\prime}(x)$ $(1 \leq k \leq l)$ satisfy the same aggregate system of $l$ differential equations with $l$ unknowns and the same upper and lower boundary constraints. The spectral decomposition method, described in Section 4, provides such an aggregate differential equation system with a unique solution. Hence,

$F_{\mathcal{H}_{k} \cap D R S(N)}(x)=F_{\mathcal{H}_{k} \cap D R S\left(N^{\prime}\right)}^{\prime}(x)(1 \leq k \leq l)$.

The following proposition demonstrates that for two LFSPNs related by

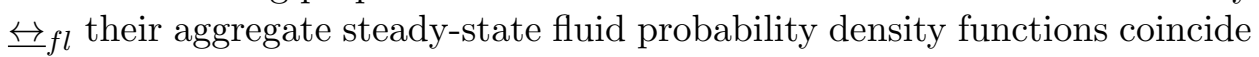
for each equivalence class of discrete markings.

Proposition 4. Let $N, N^{\prime}$ be LFSPNs with $\mathcal{R}: N_{f l} N^{\prime}$ and $f(x)=\left(f_{1}(x), \ldots, f_{n}(x)\right), n=|D R S(N)|$, be the steady-state fluid probability density function for the SFM of $N$ and $f^{\prime}(x)=\left(f_{1}^{\prime}(x), \ldots, f_{m}^{\prime}(x)\right)$, $m=\left|D R S\left(N^{\prime}\right)\right|$, be the steady-state fluid probability density function for the $S F M$ of $N^{\prime}$. Then for all $\mathcal{H} \in\left(D R S(N) \cup D R S\left(N^{\prime}\right)\right) / \mathcal{R}$ we have

$$
\sum_{\left\{i \mid M_{i} \in \mathcal{H} \cap D R S(N)\right\}} f_{i}(x)=\sum_{\left\{j \mid M_{j}^{\prime} \in \mathcal{H} \cap D R S\left(N^{\prime}\right)\right\}} f_{j}^{\prime}(x), x>0 .
$$


Proof. Remember that $f_{i}(x)=\frac{d F_{i}(x)}{d x}(1 \leq i \leq n)$ and $f_{j}^{\prime}(x)=\frac{d F_{j}^{\prime}(x)}{d x}$ $(1 \leq j \leq m)$. Let $\mathcal{H} \in\left(D R S(N) \cup D R S\left(N^{\prime}\right)\right) / \mathcal{R}$. By Proposition 3, we obtain

$$
\sum_{\left\{i \mid M_{i} \in \mathcal{H} \cap D R S(N)\right\}} F_{i}(x)=\sum_{\left\{j \mid M_{j}^{\prime} \in \mathcal{H} \cap D R S\left(N^{\prime}\right)\right\}} F_{j}^{\prime}(x), x>0 .
$$

By differentiating both sides of this equation by $x$ and applying the property for differentiating a sum, we come to

$$
\begin{aligned}
\sum_{\left\{i \mid M_{i} \in \mathcal{H} \cap D R S(N)\right\}} f_{i}(x) & =\sum_{\left\{i \mid M_{i} \in \mathcal{H} \cap D R S(N)\right\}} \frac{d F_{i}(x)}{d x}= \\
\sum_{\left\{j \mid M_{j}^{\prime} \in \mathcal{H} \cap D R S\left(N^{\prime}\right)\right\}} \frac{d F_{j}^{\prime}(x)}{d x} & =\sum_{\left\{j \mid M_{j}^{\prime} \in \mathcal{H} \cap D R S\left(N^{\prime}\right)\right\}} f_{j}^{\prime}(x), x>0 .
\end{aligned}
$$

The following proposition demonstrates that for two LFSPNs related by

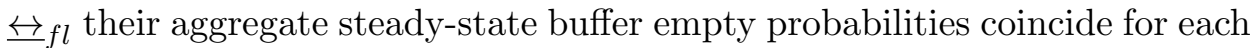
equivalence class of discrete markings.

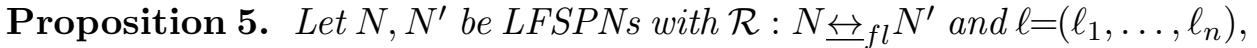
$n=|D R S(N)|$, be the steady-state buffer empty probability for the SFM of $N$ and $\ell^{\prime}(x)=\left(\ell_{1}^{\prime}, \ldots, \ell_{m}^{\prime}\right), m=\left|D R S\left(N^{\prime}\right)\right|$, be the steady-state buffer empty probability for the $S F M$ of $N^{\prime}$. Then for all $\mathcal{H} \in\left(D R S(N) \cup D R S\left(N^{\prime}\right)\right) / \mathcal{R}$ we have

$$
\sum_{\left\{i \mid M_{i} \in \mathcal{H} \cap D R S(N)\right\}} \ell_{i}=\sum_{\left\{j \mid M_{j}^{\prime} \in \mathcal{H} \cap D R S\left(N^{\prime}\right)\right\}} \ell_{j}^{\prime} .
$$

Proof. Remember that by the total probability law for the stationary behavior for the SFM of $N$, we have $\ell=\varphi-\int_{0}^{\infty} f(x) d x$.

Then for all $i(1 \leq i \leq n)$ we have $\ell_{i}=\varphi_{i}-\int_{0}^{\infty} f_{i}(x) d x$.

Let $\mathcal{H} \in\left(D R S(N) \cup D R S\left(N^{\prime}\right)\right) / \mathcal{R}$. We sum the left and right sides of the equation above for all $i$ such that $M_{i} \in \mathcal{H} \cap D R S(N)$. The result is

$$
\sum_{\left\{i \mid M_{i} \in \mathcal{H} \cap D R S(N)\right\}} \ell_{i}=\sum_{\left\{i \mid M_{i} \in \mathcal{H} \cap D R S(N)\right\}} \varphi_{i}-\sum_{\left\{i \mid M_{i} \in \mathcal{H} \cap D R S(N)\right\}} \int_{0}^{\infty} f_{i}(x) d x .
$$

Consider the right-hand side of the equation above. We apply to it the property for integrating a sum, then Proposition 2 and Proposition 4, finally, the total probability law for the stationary behavior for the SFM of $N$. Then we have $\sum_{\left\{i \mid M_{i} \in \mathcal{H} \cap D R S(N)\right\}} \ell_{i}=\sum_{\left\{i \mid M_{i} \in \mathcal{H} \cap D R S(N)\right\}} \varphi_{i}-$ $\sum_{\left\{i \mid M_{i} \in \mathcal{H} \cap D R S(N)\right\}} \int_{0}^{\infty} f_{i}(x) d x=\sum_{\left\{i \mid M_{i} \in \mathcal{H} \cap D R S(N)\right\}} \varphi_{i}-$ $\int_{0}^{\infty} \sum_{\left\{i \mid M_{i} \in \mathcal{H} \cap D R S(N)\right\}} f_{i}(x) d x=\sum_{\left\{j \mid M_{j}^{\prime} \in \mathcal{H} \cap D R S\left(N^{\prime}\right)\right\}} \varphi_{i}^{\prime}-$ $\int_{0}^{\infty} \sum_{\left\{j \mid M_{j}^{\prime} \in \mathcal{H} \cap D R S\left(N^{\prime}\right)\right\}} f_{i}^{\prime}(x) d x=\sum_{\left\{j \mid M_{j}^{\prime} \in \mathcal{H} \cap D R S\left(N^{\prime}\right)\right\}} \varphi_{i}^{\prime}-$ $\sum_{\left\{j \mid M_{j}^{\prime} \in \mathcal{H} \cap D R S\left(N^{\prime}\right)\right\}} \int_{0}^{\infty} f_{i}^{\prime}(x) d x=\sum_{\left\{j \mid M_{j}^{\prime} \in \mathcal{H} \cap D R S\left(N^{\prime}\right)\right\}} \ell_{j}^{\prime}$. 


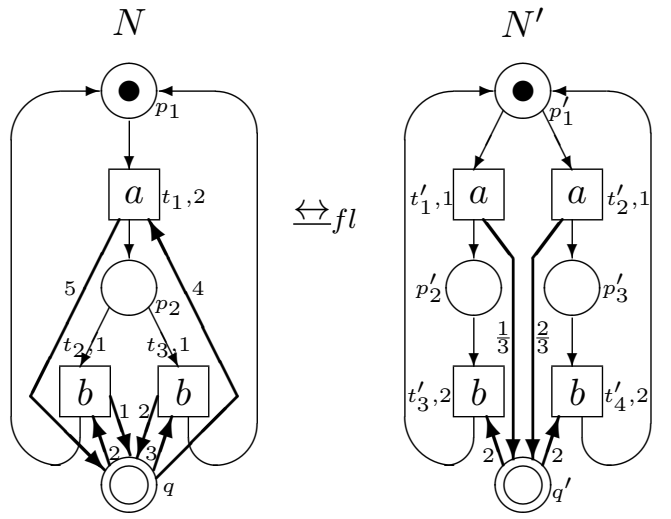

Figure 1. Fluid bisimulation equivalent LFSPNs

$D R G(N)$

$\operatorname{DRG}\left(N^{\prime}\right)$
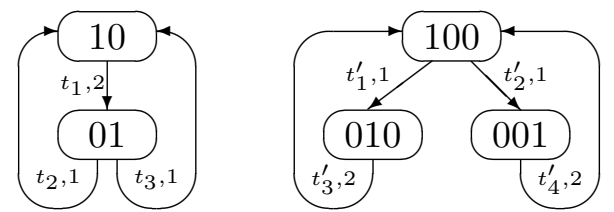

Figure 2. Discrete reachability graphs of the fluid bisimulation equivalent LFSPNs $C T M C(N)$ $C T M C\left(N^{\prime}\right)$
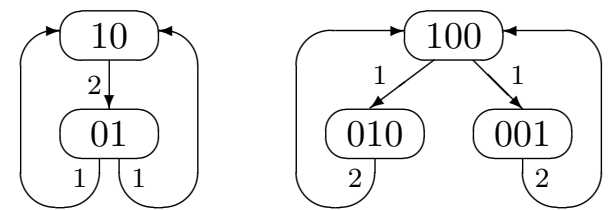

Figure 3. Underlying CTMCs of the fluid bisimulation equivalent LFSPNs

\section{Illustrative example}

Consider an example of fluid bisimulation equivalence between LFSPNs that shows how the equivalence preserves their functionality and performance.

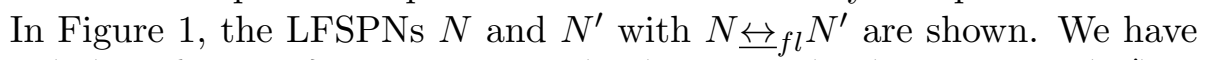
$D R G(N)=\left\{M_{1}, M_{2}\right\}$, where $M_{1}=(1,0), M_{2}=(0,1)$, and $D R G\left(N^{\prime}\right)=$ $\left\{M_{1}^{\prime}, M_{2}^{\prime}, M_{3}^{\prime}\right\}$, where $M_{1}^{\prime}=(1,0,0), M_{2}^{\prime}=(0,1,0), M_{3}^{\prime}=(0,0,1)$. In Figure 2, the discrete reachability graphs $D R G(N)$ and $D R G\left(N^{\prime}\right)$ are depicted. In Figure 3, the underlying CTMCs $C T M C(N)$ and $C T M C\left(N^{\prime}\right)$ are drawn.

We have $\operatorname{DRS}(N) /_{\mathcal{R}_{f l}(N)}=\left\{\mathcal{K}_{1}, \mathcal{K}_{2}\right\}$, where $\mathcal{K}_{1}=\left\{M_{1}\right\}, \mathcal{K}_{2}=\left\{M_{2}\right\}$, and $D R S\left(N^{\prime}\right) /_{\mathcal{R}_{f l}\left(N^{\prime}\right)}=\left\{\mathcal{K}_{1}^{\prime}, \mathcal{K}_{2}^{\prime}\right\}$, where $\mathcal{K}_{1}^{\prime}=\left\{M_{1}^{\prime}\right\}, \mathcal{K}_{2}^{\prime}=\left\{M_{2}^{\prime}, M_{3}^{\prime}\right\}$.

The sojourn time average and variance vectors of $N$ are

$$
S J=\left(\frac{1}{2}, \frac{1}{2}\right) .
$$

The TRM $\mathbf{Q}$ for $C T M C(N)$ and FRM $\mathbf{R}$ for the SFM of $N$ are 


$$
\mathbf{Q}=\left(\begin{array}{cc}
-2 & 2 \\
2 & -2
\end{array}\right), \quad \mathbf{R}=\left(\begin{array}{cc}
1 & 0 \\
0 & -2
\end{array}\right)
$$

We have $D R S^{-}(N)=\left\{M_{2}\right\}, D R S^{0}(N)=\emptyset$ and $D R S^{+}(N)=\left\{M_{1}\right\}$.

The steady-state PMF for $C T M C(N)$ is

$$
\varphi=\left(\frac{1}{2}, \frac{1}{2}\right) .
$$

Then the stability condition for the SFM of $N$ is fulfilled: FluidFlow $(q)=\sum_{i=1}^{2} \varphi_{i} R P\left(M_{i}\right)=\frac{1}{2} \cdot 1+\frac{1}{2}(-2)=-\frac{1}{2}<0$.

For each eigenvalue $\gamma$ we must have $|\gamma \mathbf{R}-\mathbf{Q}|=\left|\begin{array}{cc}\gamma+2 & -2 \\ -2 & -2 \gamma+2\end{array}\right|=$ $-2 \gamma(1+\gamma)=0$; hence, $\gamma_{1}=0$ and $\gamma_{2}=-1$.

The corresponding eigenvectors are the solutions of

$$
v_{1}\left(\begin{array}{cc}
2 & -2 \\
-2 & 2
\end{array}\right)=0, \quad v_{2}\left(\begin{array}{cc}
1 & -2 \\
-2 & 4
\end{array}\right)=0 .
$$

The (normalized) eigenvectors are $v_{1}=\left(\frac{1}{2}, \frac{1}{2}\right)$ and $v_{2}=\left(\frac{2}{3}, \frac{1}{3}\right)$.

Since $\varphi=F(\infty)=a_{1} v_{1}$, we have $F(x)=\varphi+a_{2} e^{\gamma_{2} x} v_{2}$ and $a_{1}=1$. Since $\forall M_{l} \in D R S^{+}(N) F_{l}(0)=\varphi_{l}+a_{2} v_{2 l}=0$ and $D R S^{+}(N)=\left\{M_{1}\right\}$, we have $\varphi_{1}+a_{2} v_{21}=\frac{1}{2}+a_{2} \frac{2}{3}=0$; hence, $a_{2}=-\frac{3}{4}$.

Then the steady-state fluid PDF for the SFM of $N$ is

$$
F(x)=\left(\frac{1}{2}-\frac{1}{2} e^{-x}, \frac{1}{2}-\frac{1}{4} e^{-x}\right) .
$$

The steady-state fluid probability density function for the SFM of $N$ is

$$
f(x)=\frac{d F(x)}{d x}=\left(\frac{1}{2} e^{-x}, \frac{1}{4} e^{-x}\right) .
$$

The steady-state buffer empty probability for the SFM of $N$ is

$$
\ell=F(0)=\left(0, \frac{1}{4}\right)
$$

The sojourn time average and variance vectors of $N^{\prime}$ are

$$
S J^{\prime}=\left(\frac{1}{2}, \frac{1}{2}, \frac{1}{2}\right) .
$$

The TRM $\mathbf{Q}^{\prime}$ for $C T M C\left(N^{\prime}\right)$ and FRM $\mathbf{R}^{\prime}$ for the SFM of $N^{\prime}$ are

$$
\mathbf{Q}^{\prime}=\left(\begin{array}{ccc}
-2 & 1 & 1 \\
2 & -2 & 0 \\
2 & 0 & -2
\end{array}\right), \quad \mathbf{R}^{\prime}=\left(\begin{array}{ccc}
1 & 0 & 0 \\
0 & -2 & 0 \\
0 & 0 & -2
\end{array}\right) \text {. }
$$

We have $D R S^{-}\left(N^{\prime}\right)=\left\{M_{2}^{\prime}, M_{3}^{\prime}\right\}, D R S^{0}\left(N^{\prime}\right)=\emptyset$ and $D R S^{+}\left(N^{\prime}\right)=\left\{M_{1}^{\prime}\right\}$.

The steady-state PMF for $C T M C\left(N^{\prime}\right)$ is 


$$
\varphi^{\prime}=\left(\frac{1}{2}, \frac{1}{4}, \frac{1}{4}\right)
$$

Then the stability condition for the SFM of $N^{\prime}$ is fulfilled:

FluidFlow $\left(q^{\prime}\right)=\sum_{j=1}^{3} \varphi_{j}^{\prime} R P\left(M_{j}^{\prime}\right)=\frac{1}{2} \cdot 1+\frac{1}{4}(-2)+\frac{1}{4}(-2)=-\frac{1}{2}<0$.

For each eigenvalue $\gamma^{\prime}$ we must have $\left|\gamma^{\prime} \mathbf{R}^{\prime}-\mathbf{Q}^{\prime}\right|=$

$$
\left|\begin{array}{ccc}
\gamma^{\prime}+2 & -1 & -1 \\
-2 & -2 \gamma^{\prime}+2 & 0 \\
-2 & 0 & -2 \gamma^{\prime}+2
\end{array}\right|=-2 \gamma^{\prime}\left(1+\gamma^{\prime}\right)\left(1-\gamma^{\prime}\right)=0 \text {; hence, }
$$

$\gamma_{1}^{\prime}=0, \gamma_{2}^{\prime}=-1$ and $\gamma_{3}^{\prime}=1$.

By the boundedness condition, the positive eigenvalue $\gamma_{3}^{\prime}$ and the corresponding eigenvector $v_{3}^{\prime}$ should be excluded from the solution.

The remaining corresponding eigenvectors are the solutions of

$$
v_{1}^{\prime}\left(\begin{array}{ccc}
2 & -1 & -1 \\
-2 & 2 & 0 \\
-2 & 0 & 2
\end{array}\right)=0, \quad v_{2}^{\prime}\left(\begin{array}{ccc}
1 & -1 & -1 \\
-2 & 4 & 0 \\
-2 & 0 & 4
\end{array}\right)=0 .
$$

The remaining (normalized) eigenvectors are $v_{1}^{\prime}=\left(\frac{1}{2}, \frac{1}{4}, \frac{1}{4}\right)$ and $v_{2}^{\prime}=\left(\frac{2}{3}, \frac{1}{6}, \frac{1}{6}\right)$.

Since $\varphi^{\prime}=F^{\prime}(\infty)=a_{1}^{\prime} v_{1}^{\prime}$, we have $F^{\prime}(x)=\varphi^{\prime}+a_{2}^{\prime} e^{\gamma_{2}^{\prime} x} v_{2}^{\prime}$ and $a_{1}^{\prime}=1$. Since $\forall M_{l}^{\prime} \in D R S^{+}\left(N^{\prime}\right) F_{l}^{\prime}(0)=\varphi_{l}^{\prime}+a_{2}^{\prime} v_{2 l}^{\prime}=0$ and $D R S^{+}\left(N^{\prime}\right)=\left\{M_{1}^{\prime}\right\}$, we have $\varphi_{1}^{\prime}+a_{2}^{\prime} v_{21}^{\prime}=\frac{1}{2}+a_{2}^{\prime} \frac{2}{3}=0$; hence, $a_{2}=-\frac{3}{4}$.

Then the steady-state fluid PDF for the SFM of $N^{\prime}$ is

$$
F^{\prime}(x)=\left(\frac{1}{2}-\frac{1}{2} e^{-x}, \frac{1}{4}-\frac{1}{8} e^{-x}, \frac{1}{4}-\frac{1}{8} e^{-x}\right) .
$$

The steady-state fluid probability density function for the SFM of $N^{\prime}$ is

$$
f^{\prime}(x)=\frac{d F^{\prime}(x)}{d x}=\left(\frac{1}{2} e^{-x}, \frac{1}{8} e^{-x}, \frac{1}{8} e^{-x}\right) .
$$

The steady-state buffer empty probability for the SFM of $N^{\prime}$ is

$$
\ell^{\prime}=F^{\prime}(0)=\left(0, \frac{1}{8}, \frac{1}{8}\right) .
$$

We have $\left(D R S(N) \cup D R S\left(N^{\prime}\right)\right) /_{\mathcal{R}_{f l}\left(N, N^{\prime}\right)}=\left\{\mathcal{H}_{1}, \mathcal{H}_{2}\right\}$, where $\mathcal{H}_{1}=\left\{M_{1}, M_{1}^{\prime}\right\}$ and $\mathcal{H}_{2}=\left\{M_{2}, M_{2}^{\prime}, M_{3}^{\prime}\right\}$. Let us consider the equivalence class $\mathcal{H}_{2}$.

- The aggregate steady-state probabilities for $\mathcal{H}_{2}$ coincide:

$\varphi_{\mathcal{H}_{2} \cap D R S(N)}=\sum_{\left\{i \mid M_{i} \in \mathcal{H}_{2} \cap D R S(N)\right\}} \varphi_{i}=\varphi_{2}=\frac{1}{2}=\frac{1}{4}+\frac{1}{4}=\varphi_{2}^{\prime}+\varphi_{3}^{\prime}=$ $\sum_{\left\{j \mid M_{j}^{\prime} \in \mathcal{H}_{2} \cap D R S\left(N^{\prime}\right)\right\}} \varphi_{j}^{\prime}=\varphi_{\mathcal{H}_{2} \cap D R S\left(N^{\prime}\right)}^{\prime}$.

- The aggregate steady-state buffer empty probabilities for $\mathcal{H}_{2}$ coincide: $\ell_{\mathcal{H}_{2} \cap D R S(N)}=\sum_{\left\{i \mid M_{i} \in \mathcal{H}_{2} \cap D R S(N)\right\}} \ell_{i}=\ell_{2}=\frac{1}{4}=\frac{1}{8}+\frac{1}{8}=\ell_{2}^{\prime}+\ell_{3}^{\prime}=$ $\sum_{\left\{j \mid M_{j}^{\prime} \in \mathcal{H}_{2} \cap D R S\left(N^{\prime}\right)\right\}} \ell_{j}^{\prime}=\ell_{\mathcal{H}_{2} \cap D R S\left(N^{\prime}\right)}^{\prime}$.

- The aggregate steady-state fluid PDFs for $\mathcal{H}_{2}$ coincide:

$F_{\mathcal{H}_{2} \cap D R S(N)}(x)=\sum_{\left\{i \mid M_{i} \in \mathcal{H}_{2} \cap D R S(N)\right\}} F_{i}(x)=F_{2}(x)=\frac{1}{2}-\frac{1}{4} e^{-x}=$ $\frac{1}{4}-\frac{1}{8} e^{-x}+\frac{1}{4}-\frac{1}{8} e^{-x}=F_{2}^{\prime}(x)+F_{3}^{\prime}(x)=\sum_{\left\{j \mid M_{j}^{\prime} \in \mathcal{H}_{2} \cap D R S\left(N^{\prime}\right)\right\}} F_{j}^{\prime}(x)=$ $F_{\mathcal{H}_{2} \cap D R S\left(N^{\prime}\right)}^{\prime}(x)$, where $x>0$. 
- The aggregate steady-state fluid probability density functions for $\mathcal{H}_{2}$ coincide: $f_{\mathcal{H}_{2} \cap D R S(N)}(x)=\sum_{\left\{i \mid M_{i} \in \mathcal{H}_{2} \cap D R S(N)\right\}} f_{i}(x)=f_{2}(x)=$ $\frac{1}{4} e^{-x}=\frac{1}{8} e^{-x}+\frac{1}{8} e^{-x}=f_{2}^{\prime}(x)+f_{3}^{\prime}(x)=\sum_{\left\{j \mid M_{j}^{\prime} \in \mathcal{H}_{2} \cap D R S\left(N^{\prime}\right)\right\}} f_{j}^{\prime}(x)=$ $f_{\mathcal{H}_{2} \cap D R S\left(N^{\prime}\right)}^{\prime}(x)$, where $x>0$.

Many steady-state performance indices may be aggregated to make them consistent with fluid bisimulation, with quotienting of the discrete reachability graphs and underlying CTMCs, and with the induced lumping of the

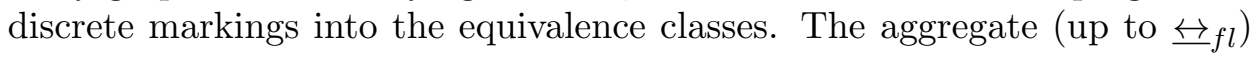
steady-state performance measures of $N$ based on the probability functions $\varphi, \ell, F(x)$ and $f(x)$ should coincide with those of $N^{\prime}$ based on $\varphi^{\prime}, \ell^{\prime}, F^{\prime}(x)$ and $f^{\prime}(x)$, respectively. Let us check this for the equivalence class $\mathcal{H}_{2}$.

- The aggregate fraction (proportion) of time spent in the set of discrete markings $\mathcal{H}_{2} \cap D R S(N)$ is TimeFract $\left(\mathcal{H}_{2} \cap D R S(N)\right)=$ $\sum_{\left\{i \mid M_{i} \in \mathcal{H}_{2} \cap D R S(N)\right\}} \varphi_{i}=\varphi_{2}=\frac{1}{2}$.

The aggregate fraction (proportion) of time spent in the set of discrete markings $\mathcal{H}_{2} \cap D R S\left(N^{\prime}\right)$ is TimeFract $\left(\mathcal{H}_{2} \cap D R S\left(N^{\prime}\right)\right)=$ $\sum_{\left\{j \mid M_{j}^{\prime} \in \mathcal{H}_{2} \cap D R S\left(N^{\prime}\right)\right\}} \varphi_{i}^{\prime}=\varphi_{2}^{\prime}+\varphi_{3}^{\prime}=\frac{1}{4}+\frac{1}{4}=\frac{1}{2}$.

- The aggregate firing frequency (throughput) of the transitions enabled in the discrete markings from $\mathcal{H}_{2} \cap D R S(N)$ is

FiringFreq $q_{\mathcal{H}_{2} \cap D R S(N)}=\sum_{t \in T_{N}}$ FiringFreq $q_{\mathcal{H}_{2} \cap D R S(N)}(t)=$ $\sum_{t \in T_{N}} \sum_{\left\{i \mid t \in \operatorname{Ena}\left(M_{i}\right), M_{i} \in \mathcal{H}_{2} \cap D R S(N)\right\}} \varphi_{i} \Omega_{N}\left(t, M_{i}\right)=$ $\varphi_{2} \Omega_{N}\left(t_{2}, M_{2}\right)+\varphi_{2} \Omega_{N}\left(t_{3}, M_{2}\right)=\frac{1}{2} \cdot 1+\frac{1}{2} \cdot 1=\frac{1}{2}+\frac{1}{2}=1$.

The aggregate firing frequency (throughput) of the transitions enabled in the discrete markings from $\mathcal{H}_{2} \cap D R S\left(N^{\prime}\right)$ is

FiringFreq $q_{\mathcal{H}_{2} \cap D R S\left(N^{\prime}\right)}=\sum_{t^{\prime} \in T_{N^{\prime}}}$ FiringFreq $\mathcal{H}_{\mathcal{H}_{2} \cap D R S\left(N^{\prime}\right)}\left(t^{\prime}\right)=$ $\sum_{t^{\prime} \in T_{N^{\prime}}} \sum_{\left\{j \mid t^{\prime} \in \operatorname{Ena}\left(M_{j}^{\prime}\right), M_{j}^{\prime} \in \mathcal{H}_{2} \cap D R S\left(N^{\prime}\right)\right\}} \varphi_{j}^{\prime} \Omega_{N^{\prime}}\left(t^{\prime}, M_{j}^{\prime}\right)=$ $\varphi_{2}^{\prime} \Omega_{N^{\prime}}\left(t_{3}^{\prime}, M_{2}^{\prime}\right)+\varphi_{3}^{\prime} \Omega_{N^{\prime}}\left(t_{4}^{\prime}, M_{3}^{\prime}\right)=\frac{1}{4} \cdot 2+\frac{1}{4} \cdot 2=\frac{1}{2}+\frac{1}{2}=1$.

- The aggregate exit frequency of the discrete markings from $\mathcal{H}_{2} \cap D R S(N)$ is $\operatorname{ExitFreq}\left(\mathcal{H}_{2} \cap D R S(N)\right)=\frac{\sum_{\left\{i \mid M_{i} \in \mathcal{H}_{2} \cap D R S(N)\right\}} \varphi_{i}}{S J\left(\mathcal{H}_{2} \cap D R S(N)\right)}=\frac{\varphi_{2}}{S J\left(M_{2}\right)}=\frac{1}{2} \cdot \frac{2}{1}=1$.

The aggregate exit frequency of the discrete markings from $\mathcal{H}_{2} \cap D R S\left(N^{\prime}\right)$ is $\operatorname{ExitFreq}\left(\mathcal{H}_{2} \cap D R S\left(N^{\prime}\right)\right)=\frac{\sum_{\left\{j \mid M_{j}^{\prime} \in \mathcal{H}_{2} \cap D R S\left(N^{\prime}\right)\right\}} \varphi_{j}^{\prime}}{S J\left(\mathcal{H}_{2} \cap D R S\left(N^{\prime}\right)\right)}=\frac{\varphi_{2}^{\prime}+\varphi_{3}^{\prime}}{S J\left(M_{2}^{\prime}\right)}=$ $\frac{\varphi_{2}^{\prime}+\varphi_{3}^{\prime}}{S J\left(M_{3}^{\prime}\right)}=\left(\frac{1}{4}+\frac{1}{4}\right) \frac{2}{1}=1$.

- The aggregate mean potential fluid flow rate for the continuous place $q$ in the discrete markings from $\mathcal{H}_{2} \cap D R S(N)$ is FluidFlow $\mathcal{H}_{2} \cap D R S(N)(q)=\sum_{\left\{i \mid M_{i} \in \mathcal{H}_{2} \cap D R S(N)\right\}} \varphi_{i} R P\left(\mathcal{H}_{2} \cap D R S(N)=\right.$ $\varphi_{2} R P\left(M_{2}\right)=\frac{1}{2}(-2)=-1$. 
The aggregate mean potential fluid flow rate for the continuous place $q$ in the discrete markings from $\mathcal{H}_{2} \cap D R S\left(N^{\prime}\right)$ is FluidFlow $_{\mathcal{H}_{2} \cap D R S\left(N^{\prime}\right)}(q)=\sum_{\left\{j \mid M_{j}^{\prime} \in \mathcal{H}_{2} \cap D R S\left(N^{\prime}\right)\right\}} \varphi_{j}^{\prime} R P\left(\mathcal{H}_{2} \cap D R S\left(N^{\prime}\right)\right)$ $=\left(\varphi_{2}^{\prime}+\varphi_{3}^{\prime}\right) R P\left(M_{2}^{\prime}\right)=\left(\varphi_{2}^{\prime}+\varphi_{3}^{\prime}\right) R P\left(M_{3}^{\prime}\right)=\left(\frac{1}{4}+\frac{1}{4}\right)(-2)=-1$.

- The aggregate traversal frequency of the move from the discrete markings from $\mathcal{H}_{2} \cap D R S(N)$ to the discrete markings from $\mathcal{H}_{1} \cap D R S(N)$ is TravFreq $\left(\mathcal{H}_{2} \cap D R S(N), \mathcal{H}_{1} \cap D R S(N)\right)=$ $\sum_{\left\{i \mid M_{i} \in \mathcal{H}_{2} \cap D R S(N)\right\}} \varphi_{i} R M\left(\mathcal{H}_{2} \cap D R S(N), \mathcal{H}_{1} \cap D R S(N)\right)=$ $\varphi_{2} R M\left(M_{2}, M_{1}\right)=\frac{1}{2} \cdot 2=1$

The aggregate traversal frequency of the move from the discrete markings from $\mathcal{H}_{2} \cap D R S\left(N^{\prime}\right)$ to the discrete markings from $\mathcal{H}_{1} \cap D R S\left(N^{\prime}\right)$ is TravFreq $\left(\mathcal{H}_{2} \cap D R S\left(N^{\prime}\right), \mathcal{H}_{1} \cap D R S\left(N^{\prime}\right)\right)=$ $\sum_{\left\{j \mid M_{j}^{\prime} \in \mathcal{H}_{2} \cap D R S\left(N^{\prime}\right)\right\}} \varphi_{j}^{\prime} R M\left(\mathcal{H}_{2} \cap D R S\left(N^{\prime}\right), \mathcal{H}_{1} \cap D R S\left(N^{\prime}\right)\right)=$ $\left(\varphi_{2}^{\prime}+\varphi_{3}^{\prime}\right) R M\left(M_{2}^{\prime}, M_{1}^{\prime}\right)=\left(\varphi_{2}^{\prime}+\varphi_{3}^{\prime}\right) R M\left(M_{3}^{\prime}, M_{1}^{\prime}\right)=\left(\frac{1}{4}+\frac{1}{4}\right) 2=1$.

- The aggregate probability of the positive fluid level in the continuous place $q$ in the discrete markings from $\mathcal{H}_{2} \cap D R S(N)$ is

FluidLevel $_{\mathcal{H}_{2} \cap D R S(N)}(q)=\sum_{\left\{i \mid M_{i} \in \mathcal{H}_{2} \cap D R S(N)\right\}}\left(\varphi_{i}-\ell_{i}\right)=\varphi_{2}-\ell_{2}=$ $\frac{1}{2}-\frac{1}{4}=\frac{1}{4}$.

The aggregate probability of the positive fluid level in the continuous place $q^{\prime}$ in the discrete markings from $\mathcal{H}_{2} \cap D R S\left(N^{\prime}\right)$ is

FluidLevel $\mathcal{H}_{2} \cap D R S\left(N^{\prime}\right)\left(q^{\prime}\right)=\sum_{\left\{j \mid M_{j}^{\prime} \in \mathcal{H}_{2} \cap D R S\left(N^{\prime}\right)\right\}}\left(\varphi_{j}^{\prime}-\ell_{j}^{\prime}\right)=$ $\left(\varphi_{2}^{\prime}-\ell_{2}^{\prime}\right)+\left(\varphi_{3}^{\prime}-\ell_{3}^{\prime}\right)=\left(\frac{1}{4}-\frac{1}{8}\right)+\left(\frac{1}{4}-\frac{1}{8}\right)=\frac{1}{8}+\frac{1}{8}=\frac{1}{4}$.

\section{Conclusion}

In this paper, we have defined fluid bisimulation equivalence for LFSPNs that preserves their qualitative and quantitative behavior, related to both their discrete part (labeled CTSPNs and the underlying CTMCs) and continuous part (the associated SFMs). We have proved that the mentioned equivalence preserves the qualitative as well as the stationary quantitative behavior and, therefore, guarantees that the functionality and performance measures of equivalent systems are identical.

In the future, we plan to define a fluid place bisimulation relation that connects "similar" continuous places of LFSPNs, like place bisimulations $[1,24,25]$ relate discrete places of Petri nets. The lifting of the relation to the discrete-continuous LFSPN markings (with discrete markings treated as the multisets of places) will respect both the fluid distribution among the related continuous places and the rates of fluid flow through them. For this purpose, we should introduce a novel notion of the multiset analogue with non-negative real-valued multiplicities of the elements. While multiset 
is a mapping from a countable set to all natural numbers, we need a more sophisticated mapping from the set of continuous places to all non-negative real numbers, corresponding to the associated fluid levels. Such an extension of the multiset notion may use the results of [4], concerning hybrid sets (the multiplicities of the elements are arbitrary integers) and fuzzy multisets (the multiplicities belong to the interval $[0 ; 1]$ ). In this way, both the initial amount of fluid and its transit flow rate in each discrete marking may be distributed among several continuous places of an LFSPN, such that all of them are bisimilar to a particular continuous place of the equivalent LFSPN. Interestingly, the fluid distributed among several bisimilar continuous places should be taken as the fluid contained in a single continuous place resulting from aggregating those "constituent" continuous places with the use of fluid place bisimulation. Then the fluid level in the "aggregate" continuous place is a sum of the fluid levels in the "constituent" continuous places. The probability density function for the sum of random variables representing the fluid levels in the "constituent" continuous places is defined via convolution. In this approach, we should avoid or treat correctly the situations when the fluid flow in the "aggregate" continuous place becomes suddenly non-continuous. This happens when some of the "constituent" continuous places are emptied while the others still contain a positive amount of fluid. Obviously, such a discontinuity is a result of applying the aggregation since it is not caused by either reaching the lower fluid boundary (zero fluid level) or change of the current discrete marking. We assume that summation of the fluid levels in the continuous places may be implemented with the constructions proposed in [11] for extended FSPNs (EFSPNs). In EFSPNs, there are special deterministic fluid jump arcs that are used to transfer a deterministic amount of fluid from one continuous place to another via intermediate stochastic transitions connecting both places (deterministic fluid transfer). Analogously, random fluid jump arcs in EFSPNs transfer a random amount of fluid from one continuous place to another (random fluid transfer).

We also intend to apply to LFSPNs an analogue of the effective reduction technique based on the place bisimulations of Petri nets [1]. We shall merge the equivalent continuous places and sometimes even the transitions between them. This usually results in the significant reductions of LFSPNs. The number of continuous places in an LFSPN impacts drastically the complexity of its solution. The analytical solution is normally possible for just a few (or for one) continuous places. Otherwise, while modeling realistic large and complex systems, we have to apply numerical techniques to solve systems of partial differential equations or the simulation method. Hence, the reduction of the number of continuous places accomplished with the place bisimulation merging is even more important for LFSPNs than for Petri nets. 


\section{References}

[1] Autant C., Schnoebelen Ph. Place bisimulations in Petri nets // Lect. Notes Comput. Sci. - 1992. - Vol. 616. - P. 45-61.

[2] Balbo G. Introduction to generalized stochastic Petri nets // Lect. Notes Comput. Sci. - 2007. - Vol. 4486. - P. 83-131.

[3] Bernardo M. A survey of Markovian behavioral equivalences // Lect. Notes Comput. Sci. - 2007. - Vol. 4486. - P. 180-219.

[4] Blizard W.D. The development of multiset theory // The Review of Modern Logic. - 1991. - Vol. 1, No. 4. - P. 319-352.

[5] Bobbio A., Puliafito A., Telek M., Trivedi K.S. Recent developments in nonMarkovian stochastic Petri nets // J. of Circuits, Systems and Computers. World Scientific, 1998. - Vol. 8, No. 1. - P. 119-158. -

http://people.ee.duke.edu/ kst/spn_papers/bobb98c.ps.

[6] Buchholz P. A notion of equivalence for stochastic Petri nets // Lect. Notes Comput. Sci. - 1995. - Vol. 935. - P. 161-180.

[7] Buchholz P. Iterative decomposition and aggregation of labeled GSPNs // Lect. Notes Comput. Sci. - 1998. - Vol. 1420. - P. 226-245.

[8] Ciardo G., Muppala J.K., Trivedi K.S. On the solution of GSPN reward models // Performance Evaluation. - 1991. - Vol. 12, No. 4. - P. 237-253. http://people.ee.duke.edu/^kst/spn_papers/gspnrew.ps.

[9] Ciardo G., Nicol D., Trivedi K.S. Discrete-Event Simulation of Fluid Stochastic Petri Nets. - Institute for Computer Applications in Science and Engineering, Hampton, Virginia, USA, 1997. - (Tech. Rep. / NASA Langley Research Center; Vol. 97-24). - http://historical.ncstrl.org/tr/pdf/icase/ TR-97-24.pdf.

[10] Ciardo G., Nicol D., Trivedi K.S. Discrete-event simulation of fluid stochastic Petri nets // IEEE Trans. on Software Engineering. - 1999. - Vol. 25, No. 2. - P. 207-217. - http://people.ee.duke.edu/ kst/spn_papers/ Discrete.pdf.

[11] Gribaudo M. Hybrid Formalism for Performance Evaluation: Theory and Applications: Thes. Ph.D. - Department of Computer Science, Univ. of Turin, Italy, 2002. - http://www.di.unito.it/ marcog/Downloads/ PhDThesis.pdf.

[12] Gribaudo M., Manini D., Sericola B., Telek M. Second order fluid models with general boundary behavior // Annals of Operations Research. - 2008. - Vol. 160. - P. 69-82.

[13] Gribaudo M., Sereno M. Simulation of fluid stochastic Petri nets // Proc. $8^{\text {th }}$ Int. Symp. on Modeling, Analysis and Simulation of Computer and Telecommunication Systems - 00 (MASCOTS'00). - San Francisco, USA, 2000. - P. 231-239. - http://www.di.unito.it/ ${ }^{\sim} \operatorname{marcog} /$ Downloads/MASCOTS00.pdf. 
[14] Gribaudo M., Sereno M., Horváth A., Bobbio A. Fluid stochastic Petri nets augmented with flush-out arcs: modelling and analysis // Discrete Event Dynamic Systems: Theory and Application. - 2001. - Vol. 11, Nos. 1-2. - P. 97-117. - http://www.di.unito.it/ marcog/Downloads/DEDS2001.pdf.

[15] Gribaudo M., Telek M. Fluid models in performance analysis // Lect. Notes Comput. Sci. - 2007. - Vol. 4486. - P. 271-317.

[16] Gribaudo M., Telek M. Stationary analysis of fluid level dependent bounded fluid models // Performance Evaluation. - 2008. - Vol. 65, Nos. 3-4. - P. 241-261. - http://webspn.hit.bme.hu/ telek/cikkek/grib07a.pdf.

[17] Haverkort B.R. Markovian models for performance and dependability evaluation // Lect. Notes Comput. Sci. - 2001. - Vol. 2090. - P. 38-83.

[18] Hayden R.A., Bradley J.T. A fluid analysis framework for a Markovian process algebra // Theor. Comput. Sci. - 2010. - Vol. 411. - P. 2260-2297.

[19] Hillston J. A Compositional Approach to Performance Modelling. - Cambridge University Press, UK, 1996. - http://www.dcs.ed.ac.uk/pepa/book.pdf.

[20] Horton G., Kulkarni V.G., Nicol D.M., Trivedi K.S. Fluid stochastic Petri nets: theory, applications, and solution techniques // European J. of Operations Research. - 1998. - Vol. 105, No. 1. - P. 184-201.

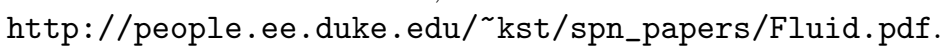

[21] Horváth A., Gribaudo M. Matrix geometric solution of fluid stochastic Petri nets // Proc. $4^{\text {th }}$ Int. Conf. on Matrix-Analytic Methods in Stochastic Models - 02. Adelaide, Australia, 2002. - P. 163-182. - http://www.di.unito.it/ marcog/Downloads/HoGr02.pdf.

[22] Marsan M.A. Stochastic Petri nets: an elementary introduction // Lect. Notes Comput. Sci. - 1990. - Vol. 424. - P. 1-29.

[23] Marsan M.A., Balbo G., Conte G., Donatelli S., Franceschinis G. Modelling with Generalised Stochastic Petri Nets. - Wiley Ser. in Parallel Comp., John Wiley and Sons, 1995. - http://www.di.unito.it/ ${ }^{\sim}$ greatspn/GSPN-Wiley.

[24] Tarasyuk I.V. Place bisimulation equivalences for design of concurrent and sequential systems // Electr. Notes Theor. Comput. Sci. - 1998. - Vol. 18. P. 191-206. - http://itar.iis.nsk.su/files/itar/pages/equpltcs.pdf.

[25] Tarasyuk I.V. $\tau$-Equivalences and Refinement for Petri Nets Based Design. Fakultät Informatik, Technische Universität Dresden, Germany, 2000. - (Tech. Berichte / Fakultät Informatik, Technische Universität Dresden; Vol. TUDFI00-11). - http://itar.iis.nsk.su/files/itar/pages/eqtbrtud_cov.pdf.

[26] Trivedi K.S., Kulkarni V.G. FSPNs: fluid stochastic Petri nets // Lect. Notes Comput. Sci. - 1993. - Vol. 691. - P. 24-31.

[27] Tschaikowski M., Tribastone M. Exact fluid lumpability for Markovian process algebra // Lect. Notes Comput. Sci. - 2012. - Vol. 7454. - P. 380-394. 
[28] Tschaikowski M., Tribastone M. Exact fluid lumpability in Markovian process algebra // Theor. Comput. Sci. - 2014. - Vol. 538. - P. 140-166.

[29] Wolter K. Second order fluid stochastic Petri nets: an extension of GSPNs for approximate and continuous modelling // Proc. Workshop on Analytical and Numerical Modelling Techniques, $1^{\text {st }}$ World Congress on Systems Simulation - 97. - Singapure, 1997. - P. 328-332. - http://page.mi.fu-berlin.de/ katinkaw/publications/anmt.ps. 
